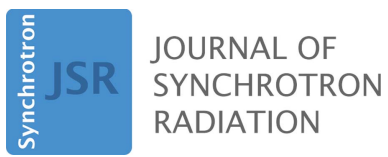

ISSN 1600-5775

Received 26 October 2020

Accepted 11 December 2020

Edited by K. Kvashnina, ESRF - The European Synchrotron, France

Keywords: macromolecular crystallography; serial crystallography; microfocus; beamlines; endstations

Supporting information: this article has supporting information at journals.iucr.org/s

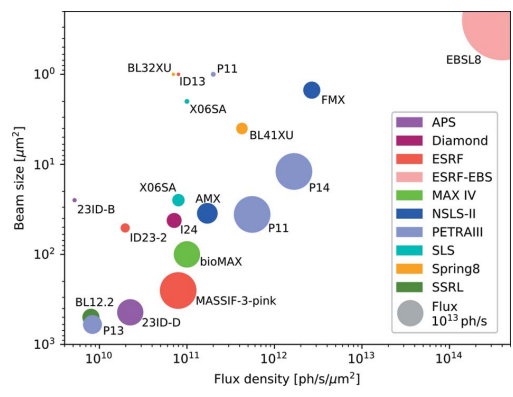

OPEN $\odot$ ACCESS

\section{FMX - the Frontier Microfocusing Macromolecular Crystallography Beamline at the National Synchrotron Light Source II}

Dieter K. Schneider, ${ }^{a}$ Wuxian Shi, ${ }^{a}$ Babak Andi, ${ }^{a}$ Jean Jakoncic, ${ }^{\text {a }}$ Yuan Gao, ${ }^{\text {a }}$ Dileep K. Bhogadi, ${ }^{b}$ Stuart F. Myers, ${ }^{\text {a Bruno Martins, }}$, John M. Skinner, a Jun Aishima, ${ }^{a}$ Kun Qian, ${ }^{a}$ Herbert J. Bernstein, ${ }^{d}$ Edwin O. Lazo, ${ }^{\text {a }}$ Thomas Langdon, ${ }^{a}$ John Lara, ${ }^{a}$ Grace Shea-McCarthy, ${ }^{a}$ Mourad Idir, ${ }^{a}$ Lei Huang, ${ }^{a}$ Oleg Chubar, ${ }^{a}$ Robert M. Sweet, ${ }^{a}$ Lonny E. Berman, ${ }^{a}$ Sean McSweeney ${ }^{a}$ and Martin R. Fuchs ${ }^{\mathrm{a} *}$

aPhoton Sciences, Brookhaven National Laboratory, Upton, NY 11973, USA, bSLAC National Accelerator Laboratory, Menlo Park, CA 94025, USA, ' Facility for Rare Isotope Beams, Michigan State University, East Lansing, MI 48824, USA, and ${ }^{\mathbf{d}}$ Ronin Institute for Independent Scholarship, c/o NSLS-II, Brookhaven National Laboratory, Upton, NY 11973, USA. *Correspondence e-mail: mfuchs@bnl.gov

Two new macromolecular crystallography (MX) beamlines at the National Synchrotron Light Source II, FMX and AMX, opened for general user operation in February 2017 [Schneider et al. (2013). J. Phys. Conf. Ser. 425, 012003; Fuchs et al. (2014). J. Phys. Conf. Ser. 493, 012021; Fuchs et al. (2016). AIP Conf. Proc. SRI2015, 1741, 030006]. FMX, the micro-focusing Frontier MX beamline in sector 17-ID-2 at NSLS-II, covers a 5-30 keV photon energy range and delivers a flux of $4.0 \times 10^{12}$ photons s$^{-1}$ at $1 \AA$ into a $1 \mu \mathrm{m} \times 1.5 \mu \mathrm{m}$ to $10 \mu \mathrm{m} \times 10 \mu \mathrm{m}(\mathrm{V} \times \mathrm{H})$ variable focus, expected to reach $5 \times 10^{12}$ photons s $^{-1}$ at final storage-ring current. This flux density surpasses most MX beamlines by nearly two orders of magnitude. The high brightness and microbeam capability of FMX are focused on solving difficult crystallographic challenges. The beamline's flexible design supports a wide range of structure determination methods - serial crystallography on micrometre-sized crystals, raster optimization of diffraction from inhomogeneous crystals, high-resolution data collection from large-unit-cell crystals, room-temperature data collection for crystals that are difficult to freeze and for studying conformational dynamics, and fully automated data collection for sample-screening and ligand-binding studies. FMX's high dose rate reduces data collection times for applications like serial crystallography to minutes rather than hours. With associated sample lifetimes as short as a few milliseconds, new rapid sample-delivery methods have been implemented, such as an ultra-high-speed high-precision piezo scanner goniometer [Gao et al. (2018). J. Synchrotron Rad. 25, 1362-1370], new microcrystal-optimized micromesh well sample holders [Guo et al. (2018). IUCrJ, 5, 238-246] and highly viscous media injectors [Weierstall et al. (2014). Nat. Commun. 5, 3309]. The new beamline pushes the frontier of synchrotron crystallography and enables users to determine structures from difficult-tocrystallize targets like membrane proteins, using previously intractable crystals of a few micrometres in size, and to obtain quality structures from irregular larger crystals.

\section{Introduction}

In recent years, microcrystallography has undergone revolutionary developments that have greatly widened its appeal for structure determination of challenging proteins (Smith et al., 2012; Owen et al., 2016; Yamamoto et al., 2017). Protein crystallographers can now obtain structures from crystals that previously would have been considered intractable, such as structures from highly inhomogeneous riboswitch crystals 
(Peselis \& Serganov, 2018) and sulfur anomalous phasing from the smallest microcrystals (Guo et al., 2019). Due to faster detectors and automation, ligand-binding studies can now explore larger libraries. One can now study large-unit-cell targets such as ribosome crystals in times previously appropriate only for lysozyme crystals.

The new Frontier Microfocusing Macromolecular Crystallography (FMX) beamline at the National Synchrotron Light Source II at Brookhaven National Laboratory (BNL) (Fuchs et al., 2014, 2016) delivers a beam of unprecedented brightness, stability and versatility, with a beam size of 1 to $10 \mu \mathrm{m}$ and a flux of $4.0 \times 10^{12}$ photons s $^{-1}$ at a wavelength of $1 \AA$. Its Eiger X $16 \mathrm{M}$ pixel-array detector supports noise-free readout at frame rates up to $750 \mathrm{~Hz}$ (in a $4 \mathrm{M}$ region of interest) for the collection of complete datasets in under one second, and data collection from hundreds to thousands of microcrystals in under one minute. Sample delivery beyond standard cryocrystallography can be tailored to the protein-crystallization environment by choosing between ultra-fast rastering serial microcrystallography, room-temperature in situ crystallography or serial crystallography in a lipidic cubic phase (LCP) injector. Automation of all crucial steps of the experiment greatly increases experiment speed: high-throughput sample-mounting robotics, high-speed raster-scan location of microcrystals, and automated data-processing pipelines supported by high-performance computing. In favorable cases, the complete process from sample mounting to structure solution is fully automated.

FMX is one of a suite of three life-science beamlines at the National Synchrotron Light Source II (NSLS-II) funded by the National Institutes of Health and by the Department of Energy's Office of Biological and Environmental Research. The two companion beamlines are LiX (Yang et al., 2020), with a focus on scattering methods, and AMX (Jakoncic et al., in preparation), with a focus on highly automated macro- molecular crystallography (MX). The design of the FMX optical system delivers a beam that brings NSLS-II's unprecedented emittance (Smaluk et al., 2019) to the crystal, and its experimental station handles this crystal with the required precision, speed and flexibility. The layout and beam properties pose specific instrumentation challenges: with a $65.7 \mathrm{~m}$ source-to-focus distance, and associated long lever arms of the optical paths, the photon-delivery system requires that the $\mathrm{X}$-ray optics be exceptionally vibrationally stable. The two MX beamlines' canted undulators share the straight section of sector 17 of NSLS-II's storage ring; this greatly limits the space for the optical components and experimental stations (see Fig. 1). The high dose rates require a reconsideration of established designs, such as the need for a sub-millisecond shutter even for shutter-less data acquisition.

\subsection{Crystallography at the one-micrometre frontier}

The FMX beamline is built to explore the frontiers of microfocus macromolecular crystallography. Its $1 \mu \mathrm{m}$ beam enables crystallographers to explore the limits of diffraction data collection at ambient pressure and under throughput conditions optimized for crystallography.

A first frontier is structure determination from the smallest crystals. If one has micrometre-scale crystals, a micrometresized beam allows one to minimize scattering from the buffer and crystal support, which would contribute to the background in the diffraction image. Simulations using fastBragg (Holton et al., 2014; Lyubimov et al., 2016), which accounts for these issues, strongly indicate that successful structure determinations from crystals of sub-micrometre sizes are possible on FMX. Indeed, we have demonstrated sulfur SAD phasing of crystals smaller than $10 \mu \mathrm{m}$ (Guo et al., 2019), and we obtained usable sub-datasets from crystals with average
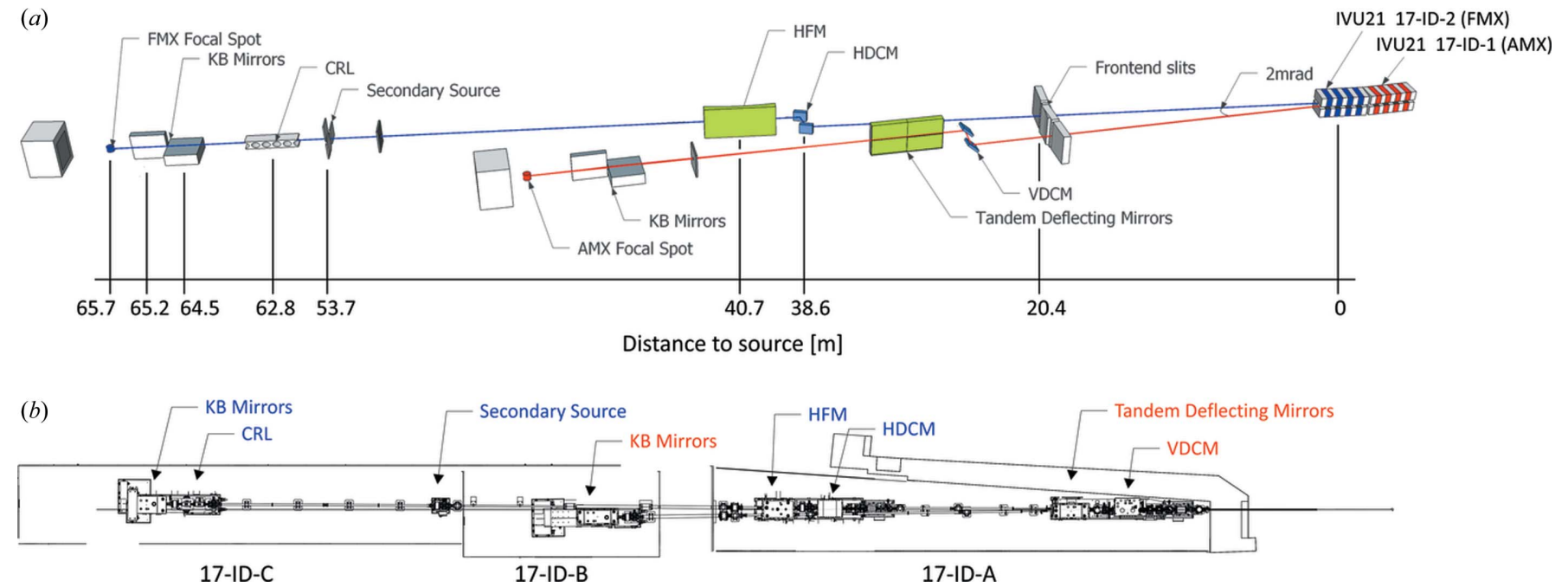

Figure 1

(a) The FMX and AMX beamline layout (not to scale). AMX is served by the upstream (17-ID-1) IVU21 undulator, FMX by the downstream (17-ID-2) one. Beam and labels of AMX are red, those of FMX in blue. All distances are given with respect to the FMX source position. (b) A top-down plan view of the beamline layout on the NSLS-II experimental floor. The white-beam path to the monochromators is contained in the 17-ID-A lead hutches, followed by the AMX experimental hutch 17-ID-B and the FMX experimental hutch 17-ID-C. 
properties for macromolecule size, cell dimensions and solvent content down to $2 \mu \mathrm{m}$ in size (unpublished data).

Data collection from the smallest microcrystals will typically not yield more than a few frames per crystal, so any complete dataset will be merged from multiple crystals. To bring new crystal targets rapidly into the beam, synchrotron serial crystallography is the method of choice (Yamamoto et al., 2017), see Section 1.1.1 below.

A second frontier at the other end of the size spectrum is the optimization of diffraction data quality from large, but irregular and inhomogeneous, crystals. Large crystals can exhibit cracks, twists and irregular growth regions, or comprise clusters of microcrystals, so a large beam would yield unusable diffraction patterns with multiple lattices or irregular reflection profiles. Rastering evaluation (Song et al., 2007; Cherezov et al., 2009; Flot et al., 2010; Aishima et al., 2010) of such a crystal with a size-optimized $1-10 \mu \mathrm{m}$ beam provides a highresolution map of the diffraction properties across the crystal's volume, and one can subsequently collect data from only the best regions (Bowler et al., 2010). We regularly encounter examples of this phenomenon where data collection from a small optimal crystal volume delivers high-quality diffraction and a complete dataset (Peselis \& Serganov, 2018). For a structural biologist tackling a challenging target, this progress in accessible crystal types means that final structures can be obtained early in the crystallization optimization cycle, thereby dramatically shortening a critical bottleneck in structure determination.

A third frontier is the reduction of radiation damage due to photoelectron escape effects. This also requires a beam size of around $1 \mu \mathrm{m}$ (Cowan \& Nave, 2008; Dickerson \& Garman, 2019; Marman et al., 2018) in the energy range applicable for macromolecular crystallography (Sanishvili et al., 2011; Finfrock et al., 2013).

For a crystallographic experiment, the beamline's high flux density translates to a high dose rate (see Section 2.6.1). The time to reach the Garman dose limit (Owen et al., 2006) can be as short as $30 \mathrm{~ms}$ for an average protein crystal on FMX. This high dose rate has three main scientific uses: a significant improvement in sample throughput for serial synchrotron crystallography (see below), the possibility for time-resolved crystallographic measurements of protein dynamics and enzyme kinetics at millisecond timescales, and the outrunning of radiation damage effects at room temperature. To realize the promise of this new performance regime, and to realize the beamline's full potential, one must establish and master new sample-handling techniques, described in the following paragraphs.

1.1.1. Serial synchrotron crystallography. The serial crystallography program on the FMX beamline supports a wide array of samples, crystallization buffers and crystal sizes and shapes to solve structures from the smallest crystals (Guo et al., 2018; Gao et al., 2018; Guo et al., 2019) and to study conformational polymorphs through statistical analysis, as demonstrated by Ebrahim et al. (2019). The associated developments range from ultrafast scanning goniometers to new sample holders and viscous injector sample delivery, from multi-crystal crystallography to free-electron-laser-style serial microcrystallography, and variable-temperature measurements from cryo- to room temperature. To open up these new methods for non-expert users we are developing easy-to-use data-processing pipelines, described in Section 6.1.

We have successfully demonstrated several dedicated serial crystallography crystal-delivery methods on the FMX beamline, such as the FastForward raster scanning goniometer (Gao et al., 2018) and in meso in situ (IMISX) LCP crystal holders (Huang et al., 2016). A high-viscosity extrusion injector (Weierstall et al., 2014) has been installed for data collection on FMX in a collaboration with the group of John Spence at Arizona State University, USA. The main appeal for the synchrotron MX user base is the optimized workflow the injector system provides for LCP-grown microcrystals. Examples from the FMX serial crystallography user program are described in Section 7.5.

1.1.2. Room-temperature and variable-temperature crystallography. The beamline's high dose rate, fast detector and fast goniometry provide new opportunities for crystallography measurements at room temperature. Secondary radiation damage effects can be outrun by high-dose-rate measurements (Warkentin et al., 2013), hard-to-freeze crystals can deliver high-quality diffraction data (Owen et al., 2017; Axford et al., 2015) and multiconformer refinement analysis of multitemperature datasets can reveal conformational variations linked to protein function that would be frozen out at cryotemperatures (Keedy et al., 2015; Russi et al., 2017).

\section{Photon delivery system}

The machine performance of the NSLS-II storage ring with a horizontal emittance of $0.8 \mathrm{~nm}$ rad (Smaluk et al., 2019) and the figure errors of the current state-of-the-art mirrors make a beam size of $1 \mu \mathrm{m}$ achievable with few compromises in available flux. We achieved this beam size with beam divergences at the sample down to $0.5 \mathrm{mrad}$, suited to the most challenging MX projects, and at working distances that still allow use of advanced goniometry and sample-delivery devices.

\subsection{Undulator}

FMX and AMX share a low-beta short straight section in sector 17 of the NSLS-II storage ring. The upstream undulator (17-ID-1) is canted outboard by $1 \mathrm{mrad}$ and delivers photons to the AMX beamline. FMX's downstream undulator (17-ID2) is canted inboard by $1 \mathrm{mrad}$ for a total canting angle of $2 \mathrm{mrad}$. At the NSLS-II design emittance of $\varepsilon_{x}=0.55 \mathrm{~nm} \mathrm{rad}$, the X-ray beam horizontal source size will be $33 \mu \mathrm{m}$ r.m.s. with an $18 \mu \mathrm{rad}$ divergence r.m.s. at $12.66 \mathrm{keV}$. At the current operational horizontal emittance of $\varepsilon_{x}=0.8 \mathrm{~nm} \mathrm{rad}$, the horizontal source size is $41 \mu \mathrm{m}$ with a divergence of $21 \mu \mathrm{rad}$. In the vertical direction, the emittance of $\varepsilon_{y}=8 \mathrm{pm}$ rad leads to a source size of $4 \mu \mathrm{m}$ with a divergence of $9 \mu \mathrm{rad}$.

Each insertion device is a $1.5 \mathrm{~m}$-long IVU21 in-vacuum undulator with Hitachi NdFeB NEOMAX magnets arranged 
with a $21 \mathrm{~mm}$ period. The minimum undulator gap under current operating conditions is $6.4 \mathrm{~mm}$, dictated by the electron-beam orbit in sector 17 , providing a peak magnetic field of $1.0 \mathrm{~T}$ and a $K$ value of 1.87 .

\subsection{Optical design}

The optical layout of FMX (Fuchs et al., 2014, 2016; Berman et al., 2011; Schneider et al., 2013) is optimized to deliver a highly stable high-flux beam into a $1 \mu \mathrm{m}$ focus, to share one short straight section of the NSLS-II storage ring with its companion beamline AMX, to support rapid beam-size changes between 1 and $10 \mu \mathrm{m}$, and to cover a photon energy range from 5 to $30 \mathrm{keV}$. The key beamline elements between the frontend and the experimental station, such as X-ray optical elements, vacuum system, slits, masks and the diagnostic screens, were designed to functional specifications. They were fabricated and installed by Bruker ASC and its successor RI Research Instruments $\mathrm{GmbH}$, Germany.

Key elements to achieving these goals are a staggering of the optical components between AMX and FMX, a pair of horizontal deflection mirrors in the AMX beam path, the use of long mirrors bent by bimorph piezoelectric transducers, a single-stage vertical and two-stage horizontal focusing scheme, and a horizontal-bounce double-crystal monochromator (HDCM) for increased stability (Fig. 1).

The first optical element after the NSLS-II shield wall is the AMX vertically deflecting double-crystal monochromator (VDCM), at $50 \mathrm{~mm}$ lateral beam separation. A tandem horizontally deflecting mirror pair downstream of the AMX monochromator at $30.8 \mathrm{~m}$ from the center of the short straight have incidence angles of $3.5 \mathrm{mrad}$ each to increase the relative angle between the beams by $14 \mathrm{mrad}$. The first optical element of FMX is the inboard-deflecting horizontal DCM (HDCM), followed by the inboard-deflecting first horizontal focusing mirror (HFM), both elements sharing a granite support block. The monochromatic FMX beam passes through the AMX experimental hutch to the horizontal secondary source in the FMX experimental hutch. Final focusing is achieved by a Kirkpatrick-Baez (KB) mirror pair close to the sample position at $65.7 \mathrm{~m}$. A set of Be compound refractive lenses (CRLs) upstream of the focusing mirrors can be used to expand the beam.

\subsection{Double-crystal monochromator}

In both beamlines, double-crystal monochromators (DCM) are the first optical element.

Beam stability considerations led us to choose a horizontalbounce monochromator for FMX. A vertical-bounce monochromator in FMX's single-stage focusing scheme faces very long optical axes that would require extremely stringent upper bounds on the maximally tolerable angular deviations due to vibrations; see the detailed explanation given by Fuchs et al. (2014). An apparent source movement from an angular deviation at the HDCM sees a smaller relative change to the larger horizontal source size compared with the vertical direction in the event that the DCM were vertical. Along with the larger angular beam divergence, this configuration provides a greater vibration tolerance at the expense of a slightly increased bandwidth and an acceptable transmission reduction of less than $10 \%$ above $7 \mathrm{keV}$ and less than $30 \%$ at $5 \mathrm{keV}$ compared with a vertical-bounce DCM. A horizontal deflection system with its vertical axis can also typically be designed more compact and resistant to ground vibrations.

To cover the photon energy range from 5 to $30 \mathrm{keV}$, the monochromator uses $\mathrm{Si}(111)$ crystals. The vertical Bragg rotation axis carries both crystals, with the first crystal at the rotation center. At $12.7 \mathrm{keV}$, taking into account the horizontal source divergence, the diffracted effective bandwidth is $3.9 \mathrm{eV}$ for a bandwidth of $3 \times 10^{-4}$, compared with the $1.65 \mathrm{eV}$ that a vertical-bounce DCM would have. The exit beam position is fixed by translating the gap between the crystals. The second crystal's length covers the beam motion upon variation of the Bragg angle, thus avoiding the need for a second translation.

Temperature control is achieved through liquid-nitrogen cooling of the copper blocks clamping the first crystal and a further copper block for the second crystal. Thermal contact of the second crystal's cooling block can be varied from direct clamping to a connection through braids (our current configuration) for better decoupling of vibrations.

\subsection{Focusing mirrors}

To provide vertical focusing, FMX has a single mirror in the vertical direction (VKB) with a 54-fold demagnification. In the horizontal direction it employs a two-stage focusing scheme with a first horizontal focusing mirror (HFM) at $40.7 \mathrm{~m}$ from the undulator with a demagnification of 3.1, focusing into a secondary source aperture (SSA) at $53.7 \mathrm{~m}$. A second horizontal mirror (HKB) to complete a KB mirror pair follows with a demagnification of 23. The SSA is a pair of horizontal and vertical slit blades. Using the horizontal slit pair, the beam size at the sample can be reduced by slitting down the horizontal secondary source size at the expense of flux.

All focusing mirrors are pre-shaped second-generation bimorph mirrors, each with 16 side-attached piezo bending elements (SESO, France). The HFM is a silicon mirror with a $700 \mathrm{~mm}$ optically active area and a cylindrical pre-shape that is bent to a meridional ellipse. Its slope error is $0.2 \mu \mathrm{rad}$ r.m.s. For the KB mirrors, we chose fused silica as a more flexible substrate material to support the required bending range. They have optical lengths of $650 \mathrm{~mm}(\mathrm{~V})$ and $500 \mathrm{~mm}(\mathrm{H})$, respectively, and are elliptically pre-shaped. The VKB mirror's slope error is $0.2 \mu \mathrm{rad}$ r.m.s. and the HKB mirror's slope error is $\sim 0.5 \mu \mathrm{rad}$ r.m.s. All three mirrors have stripes coated with $40 \mathrm{~nm}$ layers of $\mathrm{Pd}$ and $\mathrm{Pt}$, and the two horizontal focusing mirrors have an additional bare substrate stripe to provide harmonic rejection over the operating range from 5 to $30 \mathrm{keV}$, at incidence angles of $2.5 \mathrm{mrad}$. Due to the KB mirrors' length and high demagnification, the pitch range within which the beam size is unaffected is approximately $10 \mu \mathrm{rad}$ for the VKB and $20 \mu \mathrm{rad}$ for the $\mathrm{HKB}$, corresponding to $10 \mu \mathrm{m}$ at the sample. 
2.4.1. Focusing and beam-size control. One would like to control the beam size to meet the requirements of the specific data collection at hand (Nave, 2014) and to provide a spatial resolution for crystal location by diffraction-based rastering. The beam size can be increased without a significant reduction in flux by changing the bimorph mirrors' curvature to move the focus downstream. The height adjustment of the goniometer axis to follow the associated small shifts in the beam position is automated and takes under one minute.

In many data collection and rastering schemes, rapid focus changes are a higher priority than maximizing photon flux. For this mode, one can move the focus upstream of the sample within seconds by inserting CRLs upstream of the KB mirrors, with no need to reconfigure the mirrors. A CRL transfocator unit is installed upstream of the $\mathrm{KB}$ mirror pair. It has two banks of six sliders, each of which can hold up to four lenses. The current lens package consists of eight horizontally focusing and four vertically focusing cylinder paraboloid biconcave CRLs with a $200 \mu \mathrm{m}$ radius of curvature (RXOPTICS).

Lastly, the KB systems can be retracted from the beam to achieve nearly parallel beams at the expense of flux, e.g. for data collection on crystals with large unit cells. In this configuration, FMX's first horizontal focusing mirror and the CRLs allow one to collimate the beam. The associated beam offset at the sample position is $5 \mathrm{~mm}$ in the vertical and $2.5 \mathrm{~mm}$ in the horizontal direction, tracked by the beamconditioning devices and the microscope.

FMX currently delivers two beam-size options for energies from 9 to $15 \mathrm{keV}$ [user-adjustable between $1 \mu \mathrm{m} \times 1.5 \mu \mathrm{m}$ (Fig. 2) and $10 \mu \mathrm{m} \times 10 \mu \mathrm{m} \mathrm{V} \times \mathrm{H}$ ], while for other energies we adjust the curvature of the bimorph mirrors. These robust options will be upgraded to a rapid user-controlled selection of four discrete beam sizes $(1,3,5$ and $10 \mu \mathrm{m})$ over the whole energy range through an extended CRL assortment in the transfocator.

To achieve the smallest beam size one controls the mirror figure of all three bimorph bending mirrors. To obtain optimized voltage profiles for the bimorph benders, we use in situ slope error measurements by the pencil-beam method (Sutter et al., 2011, 2013). A complete optimization of one mirror takes approximately one hour, including influence function determination of all 16 electrodes and several iterative optimization steps for a total of $\sim 20$ scans.

2.4.2. Beam-size measurement. The beam size is evaluated at both image planes of the photon delivery systems - at the secondary source aperture, and at the sample position. At the SSA, the horizontal beam size of $25 \mu \mathrm{m}$ is large enough to use a YAG:Ce scintillator visualization screen. At the sample position, the scintillator's point spread function is too large to permit measurement of a $1 \mu \mathrm{m}$ beam size. In our default method, a nanowire scan, a $30 \mathrm{~nm}$ high $\mathrm{Cr}$ nanowire supported on a $\mathrm{Si}$ structure is scanned through the beam. The fluorescence intensity from the beam scattered by the $\mathrm{Cr}$ nanowire provides a signal proportional to the local beam intensity. A knife-edge scan using the edge of a $300 \mu \mathrm{m}$ thick tungsten wire was used as an independent verification of the $\mathrm{Cr}$ nanowire

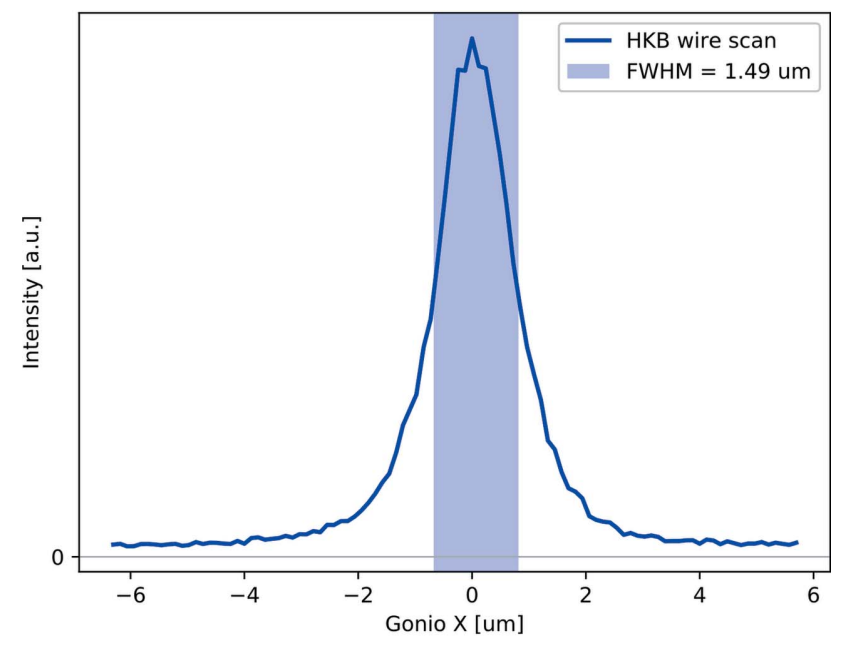

(a)

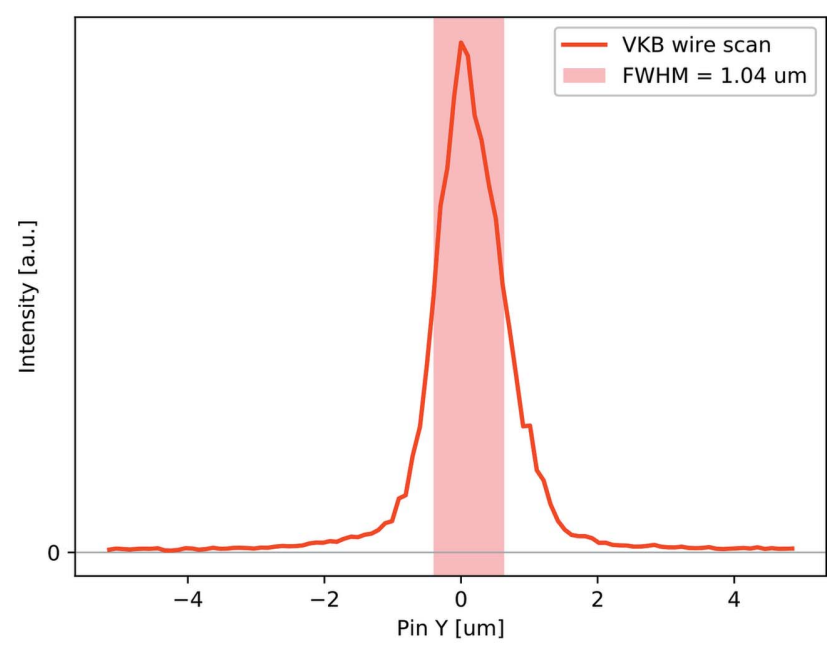

(b)

Figure 2

The FMX beam profile, showing the FMX beam spot at focus as detected by a $\mathrm{Cr}$ nanowire scan. (a) Horizontal profile with a $1.49 \mu \mathrm{m}$ FWHM. (b) Vertical profile with a $1.04 \mu \mathrm{m}$ FWHM.

scan. As a non-invasive option, the beam profile can be obtained from a histogram of the centroid offset of a pencilbeam scan profile (Sutter et al., 2011, 2013), weighted with the corresponding beam intensity at the scanned slit. At the time of writing, we have not yet commissioned this across the beamline's energy range and through a sufficiently long period, so we use the $\mathrm{Cr}$ nanowire method regularly to confirm the beam size.

\subsection{Diagnostics and feedback}

FMX employs both beam-occluding visualization screens and transmissive beam position monitors to diagnose the beam position and profile along the beamline.

2.5.1. Visualization screens. For commissioning purposes and initial local orbit adjustments, a frontend visualization flag $22 \mathrm{~m}$ from the center of the short straight section provides an undisturbed image of the photon beam at ring currents up to $2 \mathrm{~mA}$. 
For regular control of the beam alignment, one white-beam visualization screen and four monochromatic screens are placed along the beamline. The white-beam screen is a polycrystalline diamond screen upstream of the DCM, mounted at $45^{\circ}$ incidence to a water-cooled copper frame and imaged by a CCD camera (AVT Manta G-125 B) at $45^{\circ}$. Its main use is to assert the beam location in the fixed mask after longer shutdowns. The monochromatic screens are YAG:Ce scintillators inserted at normal incidence to the beam and coupled to a CCD camera (AVT Prosilica GT 2450 B) via a $90^{\circ}$ deflection mirror. They are placed downstream of the HDCM, downstream of the HFM, upstream of the SSA and upstream of the $\mathrm{KB}$ mirrors. Their use is manifold, from qualitative undulator mode and beam-shape analysis, position and flux determinations, to quantitative position scans for in situ mirror metrology.

2.5.2. Beam-position monitors. One white-beam X-ray beam-position monitor (BPM) and three monochromatic BPMs are available. The white-beam BPM is a tungsten-blade photocurrent monitor placed in the frontend upstream of the frontend slits at $15.4 \mathrm{~m}$ from the source (Ilinski, 2013). A challenge for the commissioning of this BPM is the proximity of the AMX beam $30 \mathrm{~mm}$ outboard of the FMX beam. To account for the cross-talk with the AMX beam fan, in addition to calibrating for the FMX undulator gap, the monitor was cross-calibrated to the AMX undulator gap, using a total of $10 \times 10$ gap combinations.

The monochromatic BPMs are quad-electrode CVD diamond photocurrent monitors developed at BNL (Sydor Instruments SI-DBPM-M405) (Keister et al., 2018). The first is positioned at $39.5 \mathrm{~m}$ downstream of the monochromator at $38.6 \mathrm{~m}$, the second at the secondary source at $53.1 \mathrm{~m}$, and the third at $65.5 \mathrm{~m}$ directly downstream of the focusing $\mathrm{KB}$ mirrors, $0.2 \mathrm{~m}$ upstream of the focal spot. The position resolution is $\sim 0.1 \%$ of the beam size, $100 \mathrm{~nm}$ for the BPM upstream of the sample position. All diamond monitors can be retracted from the beam to minimize absorption, e.g. to gain flux for long-wavelength experiments.

2.5.3. Encoder-based frequency analysis. Further important diagnostics for localizing and eliminating drifts and vibrations are the high-resolution encoders of the main optical elements. A kilohertz-frequency read-out with a subsequent fast Fourier transform analysis enables the correlation of critical mechanical frequencies of a specific optical element to vibrational instabilities seen in the X-ray beam.

2.5.4. Feedback stabilization. For beam position correction, three sequential feedback loops are implemented. The first steers the local electron beam orbit to keep the X-ray beam position stable on the frontend BPM. The second loop keeps the beam stable on the secondary source, and the third stabilizes the beam at the sample position.

The photon beams into AMX and FMX undergo angular drifts correlated to the $24 \mathrm{~h}$ day/night temperature cycle, due to sector 17 of the storage ring bridging the access tunnel underneath the NSLS-II storage ring. A minute-timescale bump correction, developed by the NSLS-II Accelerator Controls Group (Hidaka et al., 2019), corrects the local elec-
Table 1

Specifications of the FMX photon delivery system for the photon beam at the sample position.

\begin{tabular}{ll}
\hline Energy range & $5-30 \mathrm{keV}$ \\
Wavelength range & $0.4-2.5 \AA$ \\
Flux at focus at $12.7 \mathrm{keV}$ & $4.0 \times 10^{12}$ photons s$^{-1}$ \\
Focal spot min $(\mathrm{V} \times \mathrm{H})$ & $1 \mu \mathrm{m} \times 1.5 \mu \mathrm{m}$ \\
Focal spot range & $1-10 \mu \mathrm{m}$ \\
Divergence $(\mathrm{V} \times \mathrm{H})$ & $2 \mathrm{mrad} \times 3 \mathrm{mrad}$ to $0.5 \mathrm{mrad} \times 0.5 \mathrm{mrad}$ \\
\hline
\end{tabular}

tron orbit angle to keep the photon beam stable on the FMX frontend BPM. This reduces beam motions between the electron orbit and the beamline axis to under $20 \%$ of the beam size during an $8 \mathrm{~h}$ shift.

The beam position at the secondary source can be stabilized by a feedback loop using the roll of the first DCM crystal and the pitch of the first horizontal focusing mirror. Lastly, the beam position at the sample is stabilized via the horizontal and vertical KB mirror pitch. The two monochromatic feedback loops use the CAENels BEST system to amplify the BPM signal currents, derive position signals and control the piezo positioners for the corrective degrees of freedom. Currently, all three feedback stages are only used on drifts in the subhertz regime but may be extended to higher vibrational frequencies.

\subsection{Specifications}

Apart from its energy range, the beamline's key performance parameters are its flux and its beam size (see Table 1).

The absolute flux is checked regularly by a calibrated PIN photodiode at the sample position (see Fig. 3). For continuous flux monitoring and logging during data collection, the photocurrent measured by the CVD diamond quadrant $\mathrm{XBPM}$ is converted to photon flux and multiplied by the

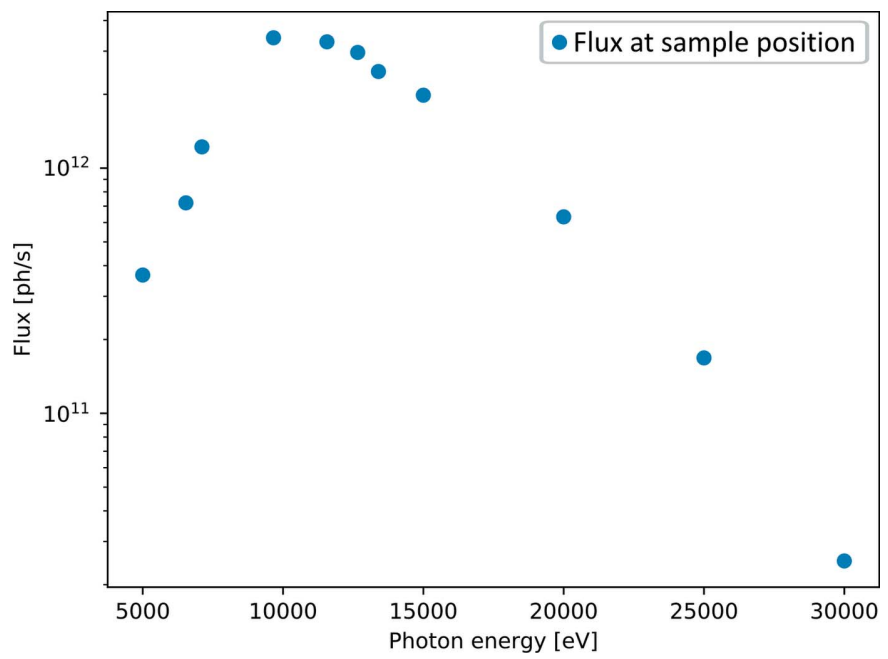

Figure 3

The FMX photon flux at the sample position across the operational photon energy range. At lower energies, the flux is diminished due to partial absorption of the horizontally polarized synchrotron radiation in the horizontal-bounce DCM, as well as by absorption in the Be exit window and the remaining air path to the sample. 


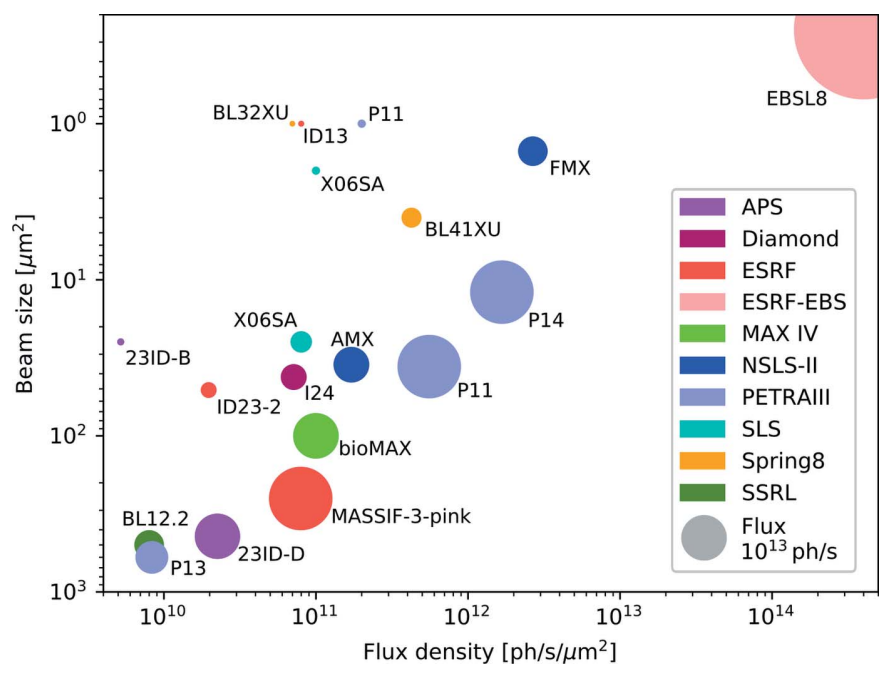

Figure 4

Flux density and beam size of FMX and selected bright microfocus MX beamlines worldwide (Allan et al., 2015; Burkhardt et al., 2016; Cianci et al., 2017; Fischetti et al., 2013; Hasegawa et al., 2013; Hirata et al., 2013; Mueller-Dieckmann et al., 2015; Riekel et al., 2010; Schulze-Briese et al., 2002; Smith et al., 2012; Soltis et al., 2008; von Stetten et al., 2020; Ursby et al., 2020). Spot size encodes maximum flux, see legend.

attenuator transmission, providing an independent alwaysonline absolute measurement.

2.6.1. Flux density comparison. Its combination of micrometre beam size and high flux places FMX into an entirely new territory of achievable flux densities. FMX surpasses the currently brightest MX beamlines by up to two orders of magnitude in achievable dose rate (Fig. 4). The design specifications of the EBSL8 beamline at the new ESRF-EBS ring are pushing this limit even higher, as indicated. The time to deliver a dose corresponding to the Garman limit (Owen et al., 2006) at a wavelength of $1 \AA$ can then be as short as $30 \mathrm{~ms}$ on FMX. This performance is a direct consequence of NSLS-II's low emittance (Smaluk et al., 2019), allowing the full flux of the insertion device to be focused into the micrometre-sized beam spot.

\section{Experimental station}

The experimental stations of FMX (Fig. 5) and AMX were designed and constructed in-house, with a focus on precision, stability and the flexibility to support a wide array of sampledelivery methods. Details of their design have been published elsewhere (Fuchs et al., 2016; Bhogadi et al., 2017) and will be summarized in an upcoming publication.

While keeping the two endstations as similar as possible, the FMX station has a secondary goniometer assembly to support specialized sample-delivery methods such as crystal jets or still (non-rotating) supports, or (not yet implemented) crystallization trays. To account for the limited space between the focusing mirror tank and the sample position of only $190 \mathrm{~mm}$ along the beam, a large granite arch around the KB mirror allows mounting of components placed further away from the beam.
The beam conditioning unit (BCU) contains (from upstream to downstream) an X-ray beam position monitor (Sydor Instruments SI-DBPM-M405) (Keister et al., 2018), an attenuator unit, an $X Y$ pair of slits, an intensity monitor, a photon shutter (Goetze \& Lienert, 2009) and a second pair of $X Y$ slits (Fuchs et al., 2016). The overall length of the BCU along the beam is only $140 \mathrm{~mm}$, facilitated by the use of an inert-gas-containing vessel rather than a bulkier and lessaccessible vacuum enclosure.

From the BCU exit window to the sample, the beam traverses the drilled mirror of the on-axis microscope (see below) and a $350 \mu \mathrm{m}$ inner diameter (ID) molybdenum tube to collimate the beam and attenuate air scatter. The $0.6 \mathrm{~mm}$ outer diameter (OD) of the tungsten beamstop corresponds to a lowest recordable resolution of $32 \AA$ at a beamstop-tosample distance of $10 \mathrm{~mm}$ and an energy of $12.7 \mathrm{keV}$. For lower-energy experiments, smaller diameter beamstops with $0.45 \mathrm{~mm}$ OD can be mounted manually on a magnetic precision mount. The alignment routine with the $X Y Z$ translations uses both the sample microscope and the flux measurement diode and takes under $5 \mathrm{~min}$.

On a second retractable positioner, a photodiode and a $\mathrm{CdWO}_{4}$ scintillator provide beam shape, position and intensity diagnostics at the focal spot (Bhogadi et al., 2017).

The on-axis microscope integrates a customized Questar QM100 telescope with a $100-150 \mathrm{~mm}$ working distance by deflecting the optical path downwards with a drilled $45^{\circ}$ mirror. It provides four always-on fixed magnifications, with a $190 \times 150 \mu \mathrm{m}$ field of view at the highest magnification to resolve crystals down to a size of $1 \mu \mathrm{m}$.

The main FMX goniometer (Bhogadi et al., 2017) is built around a Nelson Air SP150 rotary air bearing for the horizontal rotation axis and a stick-slip piezo $Y Z$ stage (SmarAct SLC-1720) for sample centering and scanning translations perpendicular to the rotation axis. The sphere of confusion at the sample position of the uncorrected goniometer axis was measured at $<500 \mathrm{~nm}$ peak to peak for a full rotation. After retracting the main goniometer inboard, the secondary goniometer can be inserted into the sample position for specialized experiments such as microcrystal jets (Weierstall et al., 2014) (Fig. 11).

With the extreme dose rates of over $250 \mathrm{MGy} \mathrm{s}^{-1}$ that the beamline can deliver, and considering the maximum frame rate of the Eiger X $16 \mathrm{M}$ of $750 \mathrm{~Hz}$ for a $4 \mathrm{M}$ region of interest (ROI), the current main goniometer is still speed-limited for large raster scans, e.g. in raster-scanning serial crystallography. To provide the positioning speed and precision to cope with these experiment conditions, we have developed a full $X Y Z$ piezo positioner-based high-speed high-precision goniometer to support operation at the full flux of FMX (Gao et al., 2018). Details of these experiments are described in Section 7.5.1 below.

To obtain X-ray fluorescence spectra and absorption edge scans, we installed a Ketek VIAMP KC Si drift detector with a $50 \mathrm{~mm}^{2}$ collimated area and an energy resolution $<133 \mathrm{eV}$.

For sample temperature control, a CryoStream cold-gas flow system (Oxford Cryosystems Ltd) covers a user-select- 
able temperature range from 80 to $400 \mathrm{~K}$. A mobile HC-Lab Humidity Controller (Arinax) can be put in place of the CryoStream system for windowless collection from crystals at room temperature.

\subsection{Area detector}

FMX's detector is a Dectris Eiger X 16M, a hybrid pixelarray single-photon-counting detector. Its central $4 \mathrm{M}$ pixels can be read out in a dedicated ROI mode with frame rates up to $750 \mathrm{~Hz}$. It has $4150 \times 4371$ pixels with a $75 \mu \mathrm{m} \times 75 \mu \mathrm{m}$ size. These specifications make it an ideal detector for highflux high-frame-rate operation, for microcrystallography, and for obtaining high-resolution datasets from large-unit-cell crystals.

The vendor guarantees a maximum sustained frame rate for a $30 \mathrm{~s}$ buffer time. We have not hit this limit in operation, because at high frame rates microcrystals yield sparse diffraction images, which compress well.

The detector support's vertical lift stage has a -100 to $+400 \mathrm{~mm}$ travel range for vertical offset measurements. With a horizontal $X$ stage, the detector can be moved outboard past the goniometer spindle for a minimal detector distance of $80 \mathrm{~mm}$. The maximum detector distance is $2.3 \mathrm{~m}$.

For data collection at long wavelengths, an He path for a fixed detector distance of $137 \mathrm{~mm}$ can be fixed mounted onto the detector, with an $8 \mu \mathrm{m}$ Kapton window $12 \mathrm{~mm}$ down- stream of the sample. It minimizes absorption of the radiation diffracted from the crystal.

\section{Automation}

FMX is equipped with a robotic sample-changer system based on an industrial six-axis robot arm (Stäubli TX60) (Lazo et al., 2021). A three-prong collet gripper developed in-house supports a SPINE sample-holder (Cipriani et al., 2006) mounting, with a planned extension to miniSPINE holders (Papp et al., 2017). The gripper can be switched automatically for different tools such as a future miniSPINE holder or crystallization-plate grippers. The liquid-nitrogen storage Dewar (Absolut System) provides space for 24 Unipucks (https://smb.slac.stanford.edu/robosync/Universal_Puck/) for a maximum capacity of 384 samples. The system is equipped with sensors at all steps of the mounting and unmounting process for sample tracking. A force/torque sensor from ATI Industrial Automation allows for calibrating and testing of the robot alignment. Eddy-current distance sensors on the endstation frame allow for testing of the robot alignment in the endstation coordinate system. An Arinax Smart Magnet system provides feedback of sample presence on the goniometer. This system has successfully mounted over 3000 user samples per cycle with a mounting reliability of over $99.8 \%$ in cycles 2019-2 and 2019-3, with a sample exchange time of $40 \mathrm{~s}$.
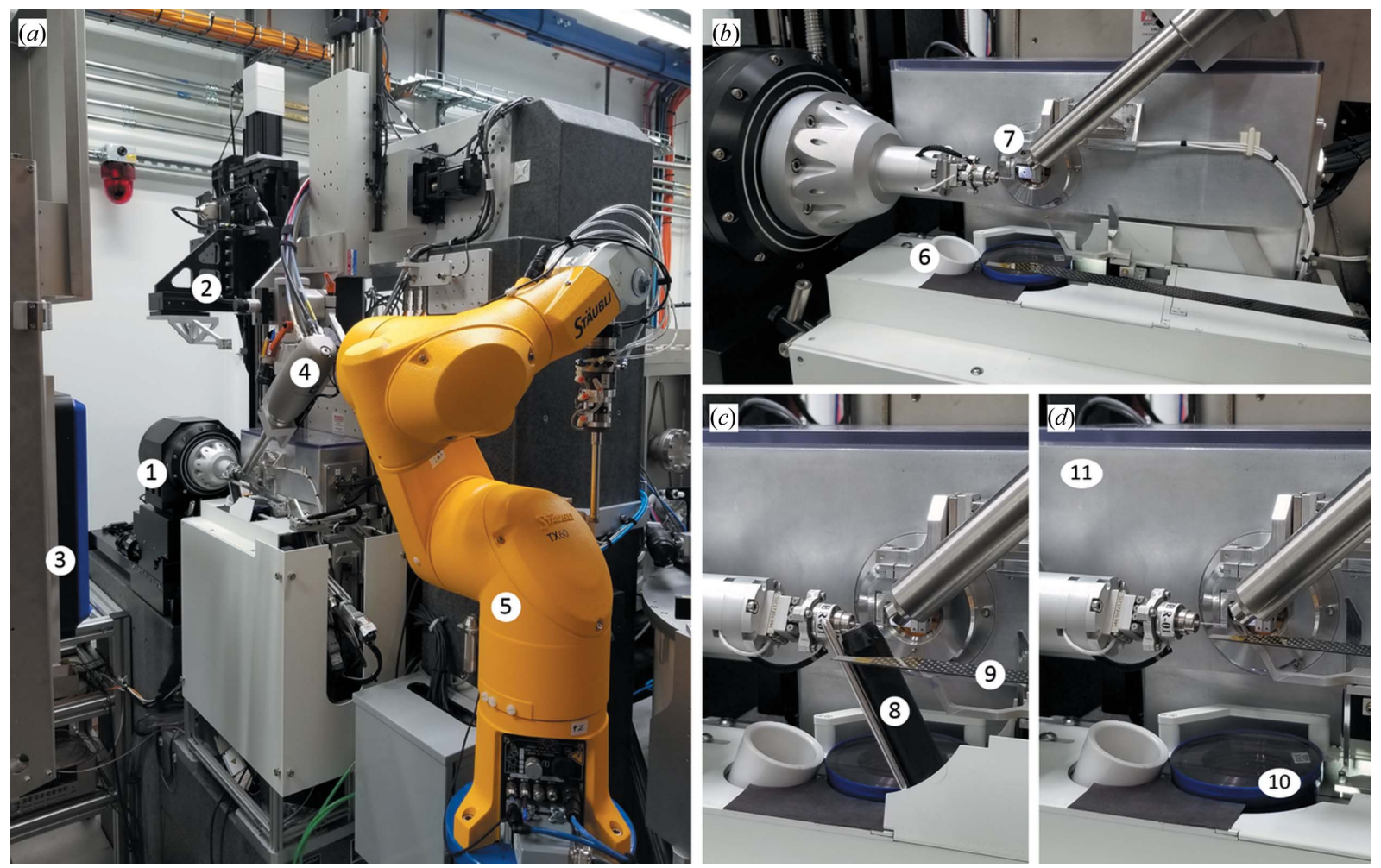

Figure 5

The FMX experimental station. (1) Main goniometer. (2) Secondary goniometer. (3) Eiger X 16M detector. (4) CryoStream CS800. (5) Samplemounting robot. (6) Cooling-gas exhaust tube. (7) Microscope deflection mirror. (8) Sample back-illumination. (9) Beam stop. (10) Questar QM100 microscope objective. (11) Beam-conditioning unit. Panels $(b)$ to $(d)$ show three experiment states of the Governor state controller (see Section 5.2) - $(b)$ sample exchange, $(c)$ sample alignment and $(d)$ data acquisition. 
AMX and FMX support a fully automated data collection mode. For crystals with sizes of $20 \mu \mathrm{m}$ or more, which are mounted in a loop that is matched to the crystal size, a mode based on auto-loop centering achieves a throughput of $>25$ samples per hour, corresponding to $\sim 130$ samples per shift, including the setup and switchover times. For other samples, we employ X-ray diffraction-based crystal centering, with a throughput of 15 samples per hour and $\sim 90$ samples per shift. In both modes, all data are collected with a pre-determined oscillation range. Further details are described in the AMX beamline paper (Jakoncic et al., in preparation).

\section{Controls systems}

The beamline's photon delivery system and endstation hardware are controlled through the Experimental Physics and Industrial Control System (EPICS, http://www.aps.anl.gov/ epics/), using Control System Studio (CSS) (Clausen et al., 2007) to provide a graphical interface. The motor controllers are DeltaTau TurboPMAC and PowerPMAC controllers. Most of the sub-micrometre-precision positioners for the slits and endstation devices are SmarAct stick-slip piezo positioners, which are controlled from the DeltaTau controllers through the SDC2 step and direction interface. All motors are operated in closed-loop mode by default. Encoder capture-based triggering is realized through Quantum Detectors Zebra boxes - one each for the slit scans of the first- and secondstage focusing mirrors, and one for the goniometer-detector synchronization.

Higher-level automation is realized through Python routines based on the NSLS-II standard Ophyd hardware abstraction layer and Bluesky data collection suite (Arkilic et al., 2017; NSLS-II, 2019). The data collection graphical user interface Life Science Data Collection (LSDC) uses both Bluesky/Ophyd and dedicated Python libraries (Fig. 6).

\subsection{Beamline optics control and automation}

To automate energy changes and beam alignment, the IVU21 undulator spectrum was mapped across the full energy range from 5 to $30 \mathrm{keV}$. In operation, a narrow undulator gap scan around the reference position is performed to maximize intensity. Correction of the pitch of the second monochromator crystal finds the peak of the DCM rocking curve around the look-up values to optimize the monochromatic flux. Then all mirrors are aligned to the energy-optimized coatings based on lookup tables, with optional position corrections based on the two monochromatic feedback loops into the secondary source and into the endstation. At the sample position, a scintillator screen is raised into the sample position and the beam center determined in the camera field of view. The height of the goniometer rotation axis is then updated to ensure its intersection with the $\mathrm{X}$-ray beam. This procedure allows users to change energies at any time during their shift without staff support.

Visualization of the rotation axis is achieved by centering a precision needle on the goniometer. This step is still

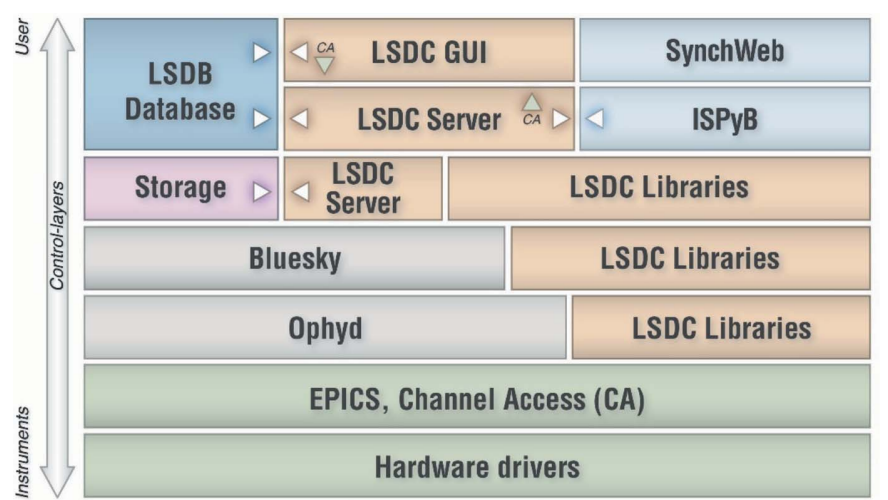

Figure 6

The controls architecture of the FMX and AMX beamlines. Communication between the different databases, servers and controllers is indicated by white arrows. The $L S D C$ GUI and server communicate through EPICS Channel Access, indicated by green arrows.

performed manually by staff, typically at the start of a user shift, but an automated routine including robotic pin mounting is currently being commissioned.

\subsection{Endstation control}

Control of the endstation configuration is the job of an experiment state manager, an EPICS input/output controller (IOC) called 'the Governor'. It provides pre-defined movement trajectories between typical experiment configuration states such as 'sample exchange' for robot access, 'sample alignment' for crystal centering, 'data acquisition' for sample exposures, or 'beam location' for beam diagnostics with a scintillator screen [see Figs. 5(a)-5(d)]. For transitions between states, where allowed, a collision-free sequence of motor movements is pre-defined, that is then executed by the Governor. Beamline applications request endstation transitions from the Governor through EPICS channel access. To speed up transitions, motors that cannot collide can be grouped to move concurrently. Within each state, each motor can be assigned an arbitrary safe movement range. If a defined safe travel range is violated by an external application, the Governor exits into a 'Maintenance' state and reports the error to facilitate troubleshooting. Limiting all position changes to one tool greatly increases the robustness of operation by eliminating accidental device collisions and providing a single reporter for positioner problems during operation.

\subsection{Data collection graphical user interface}

The data collection control software on FMX and AMX is $L S D C$. The layout of its PyQt5 graphical user interface (GUI) is based on MXCuBE2 (Gabadinho et al., 2010). It is functionally divided into three main areas: sample queue, data collection parameters and sample viewing (Fig. 7). The sample queue area on the left lists the samples stored in the automounter Dewar, as well as data collection queues and histories for each sample. Below the sample information window are command keys for the automounter, and queue management 


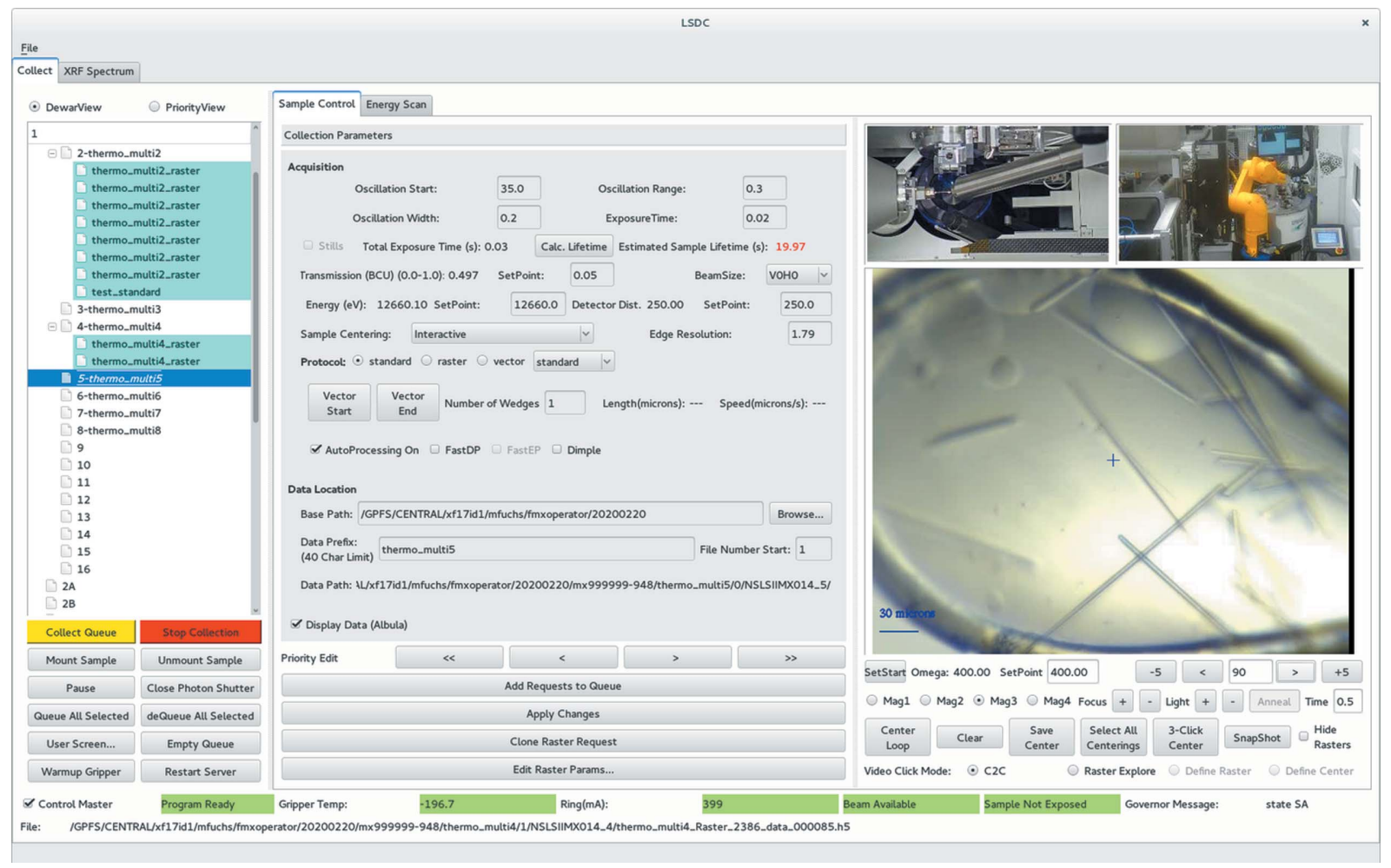

Figure 7

The LSDC GUI for AMX and FMX. The sample requests view is displayed in the left-hand column, data acquisition parameters are set in the center, and the sample video view is displayed in the right-hand column.

and execution. The data collection area in the middle of the GUI covers general parameters such as starting angle and collection range, as well as beam parameters like energy and transmission. Below are entry fields for dedicated sample centering and data collection protocols. Centering protocols can be 'Interactive' for user-control and 'Automated' for unsupervised data collection. Collection protocols include 'Standard' for taking a number of consecutive rotation images, 'Screen' for two exposures $90^{\circ}$ apart, 'Raster' for raster grid scans in a user-defined area and step size, 'Vector' for continuous or stepwise helical data collection between two points, and 'Characterization' for indexing and strategizing using EDNA (Incardona et al., 2009). Automated data processing and structure determination pipelines can be selected here as well, see below. The sample viewing area on the right is for sample inspection and centering. Four fixed magnification levels are available for visualizing crystals of different sizes. The 'Raster Explore' tool is used to check diffraction patterns based on the 'diffraction heat map' produced by raster scans. The Dectris Albula diffraction viewer is run as a separate application, interfaced for image display from $L S D C$. In a separate tab for absorption-edge scans, users select the anomalous scatterer to set the energy scan parameters, and obtain a live plot of the X-ray fluorescence signal versus beam energy and a Chooch plot (Evans \& Pettifer, 2001) displaying the inflection and peak energies, $f^{\prime}$ and $f^{\prime \prime}$. For remote data collection, users connect to the local network through the Brookaven National Laboratory virtual private network and control a dedicated data collection workstation through a NoMachine server.

\section{Data processing}

\subsection{Data reduction and structure determination}

FMX and AMX provide users with a data reduction pipeline, fastDP (Winter \& McAuley, 2011), a Python script originally from Diamond Light Source (UK) which uses $X D S$ (Kabsch, 2010), CCP4 (Winn et al., 2011) and CCTBX (Grosse-Kunstleve et al., 2002) to process data quickly. In the fast $D P$ pipeline, diffraction images are indexed and integrated in $P 1$ by $X D S$, a point group is determined through analysis from POINTLESS (Evans, 2006) and the CORRECT step in $X D S$, and finally the data are scaled in $X D S$ in the point group and merged with AIMLESS (Evans \& Murshudov, 2013). The companion script for novel structural determination, fast $E P$ (https://github.com/DiamondLightSource/fast_ep), utilizes SHELX (Sheldrick, 2015) in a brute-force structure determination approach by testing all possible space groups based on the point group from fast $D P$, and a wide range of solvent content. We developed two additional variants of fast $E P$, Fast_ep_weak and Fast_ep_NSLS2, with optimizations for experimental phasing with weak signals and for single-crystal SAD phasing, respectively. For ligand- and drug-screening experiments, a molecular replacement and ligand-visualization pipeline, DIMPLE (Winn et al., 2011), available through 
the CCP4 package (Winn et al., 2011), is also incorporated in the $L S D C$ GUI. Users provide structure models, and enter structural and sequence information associated with each crystal in a sample information spreadsheet.

Other crystallographic software available includes DOZOR (Zander et al., 2015) and DIALS spotfinder (Sauter et al., 2013) for spot finding in raster scans and serial crystallography data processing, DIALS (Winter et al., 2018) and HKL2000 (Otwinowski \& Minor, 1997) for data reduction, SHELX (Sheldrick, 2015) and HKL2MAP (Pape \& Schneider, 2004) for novel structure determination, and CCP4 (Winn et al., 2011) and PHENIX (Adams et al., 2010) as multi-purpose tools.

The data generated by the different serial crystallography methods differ greatly in how they have to be processed, e.g. still images versus $\omega$ rotation images, continuous scans versus fixed-target step rasters or step-scan vectors, data from a few crystals versus data from thousands of crystals, and also data that have a sizeable fraction of empty frames. The main building blocks of the serial crystallography processing pipelines are the multi-crystal data processing pipeline PyMDA (Guo et al., 2018; Takemaru et al., 2020), the serial crystallography pipeline called WYpeline developed for our ultra-fast raster scanning work (Gao et al., 2018), and tools for processing datasets consisting of still image exposures in jet serial crystallography like CrystFEL (White et al., 2016). A key step in all of these are clustering algorithms using one or several different metrics to choose the sub-datasets to merge for the final dataset, and various spotfinder tools (Winter et al., 2018; Zander et al., 2015) to discern images containing usable diffraction data from empty ones.

Users' sample information is managed in $I S P y B$ (Delagenière et al., 2011) and data collection and processing results are displayed through SynchWeb (Fisher et al., 2015).

\subsection{Computing}

For computing needs during user operation, AMX and FMX operate over 21 dedicated cluster nodes with 716 cores in the NSLS-II shared computing center, employing SSH calls for running distributed code and pipelines for rastering, data reduction and structure solving. For short-term storage, the two MX beamlines write data to solid-state drive storage appliances that are connected to our central high-performance General Parallel File System (GPFS) using low-latency 56 GB InfiniBand. This bandwidth is required for real-time feedback during diffraction raster scans and data reduction. For longer term storage, after $24 \mathrm{~h}$ files are transparently moved to spinning disks. The entire computing system configuration is described in greater detail in the AMX beamline article (Jakoncic et al., in preparation).

\section{User research}

\subsection{Microbeam crystallography}

In the first two years of user operation, we worked with our users to develop optimized work flows for FMX and for

\section{Beam > crystal}

- Noise from buffer scattering

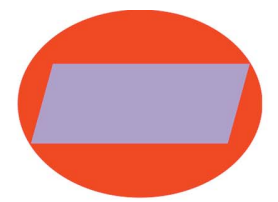

\section{Beam $=$ crystal}

- Average over crystal volume

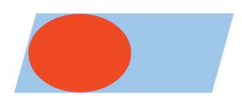

\section{Beam < crystal}

Raster-sample whole cross section

- Exclude lower-quality volume

- Separate merging of different datasets from statistically different clusters

$1 \mu \mathrm{m}$ beam: collect smallest crystals

- Serial crystallography

- Photoelectron escape reduces radiation damage

Figure 8

The benefits of microfocus crystallography, or why a micrometre-sized beam is also beneficial for data collection on larger crystals.

AMX's microbeam to realize the benefits of microfocus crystallography and make the workflows performant and intuitive to use. Data collection protocols for raster scanning a sample across the beam to locate small or obscured crystals, and well diffracting areas in larger crystals, as well as vector- or helical-scan data collection to distribute the dose across the crystal volume, have become indispensable tools (Miller et al., 2019).

As an example, large crystals can exhibit cracks, twists or irregular growth regions, which will yield unusable diffraction patterns with multiple lattices or irregular reflection profiles when illuminated with a large beam profile. A raster-scanning evaluation of such a crystal with a microfocus beam provides a high-resolution map of the diffraction properties across the crystal's volume. Data collection from a small optimal crystal volume can then deliver high-quality diffraction and a complete dataset. Once the crystals approach the size of the microfocus beam, multi-crystal data collection schemes are required to collect complete datasets, and photoelectron escape effects become efficient (Sanishvili et al., 2011; Dickerson \& Garman, 2019) (Fig. 8).

\subsection{Riboswitches}

The structure determination of riboswitches published by Peselis \& Serganov (2018) depended critically on the FMX microbeam to detect small areas within the large RNA crystal to obtain indexable diffraction patterns. Because RNA is inherently unstable and lacks hydrophobic surfaces that aid crystal packing, it often yields poorly diffracting and highly mosaic crystals that are unsuitable for data collection. Most often, RNA crystals grow as assemblies, or as macrocrystals composed of aligned, merged, small crystals. Furthermore, the RNA structure is stabilized by metal cations, which increase the sensitivity of the crystals' RNA to radiation damage. 


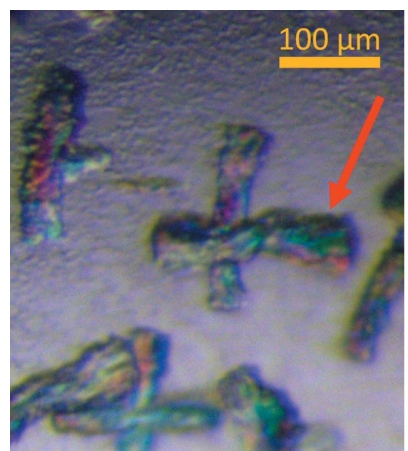

(a)

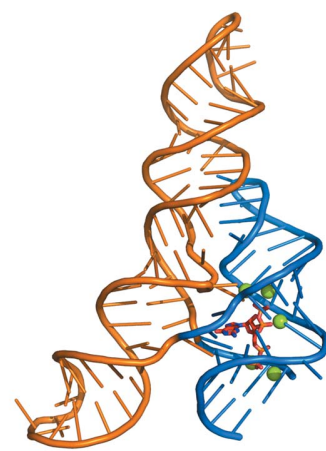

(b)
Figure 9

(a) X-shaped crystals of the ppGpp riboswitch. A red arrow shows the tip of the crystal used for collecting data on FMX. (b) The crystal structure of the ppGpp-bound riboswitch (Peselis \& Serganov, 2018). The RNA is in cartoon representation, with the sugar-phosphate backbone depicted as a cylindrical ribbon, colored according to its structural elements. ppGpp is shown as a stick model, surrounded by $\mathrm{Mg}^{2+}$ ions.

Combining FMX's fully focused beam with a vector data collection strategy allowed the collection of a full dataset from a small crystal without significant radiation damage by collecting data from only an edge of the X-shaped crystal assembly (Fig. 9).

\subsection{Sulfur phasing of Ric $8 \mathrm{~A}$}

Ric 8A, a G-protein chaperone and guanine nucleotide exchange factor, is essential for cell survival. The Sprang laboratory (University of Montana, USA) determined the structure of a major 452-residue Ric 8A domain with $\mathrm{G} \alpha$ binding activity (Zeng et al., 2019). The structure, together with further biochemical data, provides clues to the mechanism by which Ric $8 \mathrm{~A}$ interacts with its $\mathrm{G} \alpha$ substrate. Needle-like Ric 8A crystals, measuring on average $10 \mu \mathrm{m} \times 10 \mu \mathrm{m} \times 150 \mu \mathrm{m}$ in size, diffracted to $\sim 3 \AA$ at conventional synchrotron sources. There are no known homologs of Ric $8 \mathrm{~A}$, which prevents the use of molecular replacement. Further, Se-Met Ric 8A forms only microcrystals, and native Ric $8 \mathrm{~A}$ crystals are highly sensitive to heavy metals. Therefore, to provide phases for the crystal structure, the group chose to use the anomalous signal from the nine Cys and ten Met residues of the native protein to phase the structure. Multiple datasets from randomly oriented crystals were collected at $100 \mathrm{~K}$ at a wavelength of $1.77 \AA$, with a total rotation per dataset ranging from 360 to $5760^{\circ}$. The final S SAD dataset was merged from 14 crystals (11 million reflections, $\sim 800$-fold multiplicity), revealing an $\mathrm{S}$ anomalous signal significant to $3.4 \AA$. This led to a refined model for Ric $8 \mathrm{~A}$ at $2.2 \AA$ resolution. Fig. 10 shows an anomalous difference map for the sulfur substructure consisting of 40 sulfur sites, corresponding to $36 \mathrm{Met} / \mathrm{Cys}$ residues from the two Ric $8 \mathrm{~A}$ molecules and four sulfate ions in the asymmetric unit.

In a follow-up experiment, the Sprang group recently solved the Ric 8A: G $\alpha$ complex structure with data collected on FMX (McClelland et al., 2020). The plate-shaped crystals of the

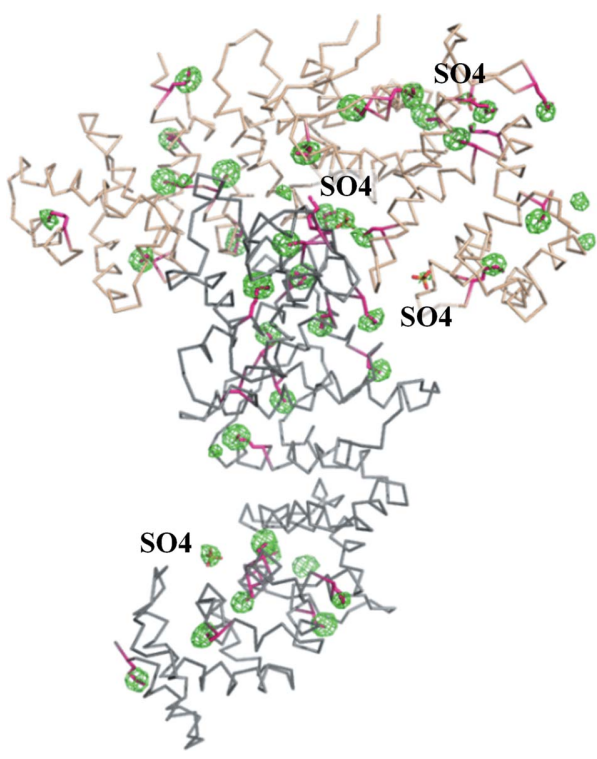

Figure 10

A structure model of Ric 8A. Anomalous difference electron-density map showing the 40 sulfur sites comprising the sulfur atom substructure in the asymmetric unit (see text), contoured at $4.5 \sigma$.

Ric 8A:G $\alpha$ :nanobody complex have an average size of $50 \mu \mathrm{m} \times 50 \mu \mathrm{m} \times 5 \mu \mathrm{m}$. These crystals diffracted weakly and anisotropically and required extensive screening. The data were filtered through STARANISO and the final structure was refined to $3.3 \AA$ resolution along the best diffracting axis. The Ric 8A: G $\alpha$ interaction surface is extensive and involves most of the ARM/HEAT of Ric 8A and its C-terminal domain. Ric 8A induces larger conformational changes in $\mathrm{G} \alpha$ than GPCRs, with a major displacement of the $\mathrm{G} \alpha \mathrm{C}$-terminus and Switch II. The new X-ray crystallography structure is overall similar to and corresponds well with a $3.9 \AA$ cryo-electron microscopy structure of this complex determined by the same group.

\subsection{Multiple-crystal data collection}

For crystals in the $1-10 \mu \mathrm{m}$ size range, radiation damage makes collection of a complete dataset from a single crystal challenging to impossible. Therefore, one often requires a multiple-crystal data collection strategy to achieve a complete dataset to the highest achievable resolution. Multiple crystals can be mounted by scooping through crystal slurries using a regular nylon loop or a mesh-type loop. Mesh loops have the advantage that it is easy to remove excess liquid before flashfreezing by application of filter paper to the back side of the mesh. Raster-scanning tools in $L S D C$, typically with a raster step size smaller than the crystal size, reveal 'hot spots' in crystals, detected by a spotfinder, suggesting positions for diffraction data collection. The software can save and queue positions that demonstrate diffraction beyond a set resolution limit for automated data collection. A typical data collection strategy might be to rotate each crystal by $5^{\circ}$ using $0.2^{\circ}$ per image and 50 images per second (500 ms total exposure per sample). Using this protocol, it takes about one second to move from crystal to crystal. 
Liu and co-workers at BNL have developed optimized micromesh sample holders and a three-step automated dataprocessing procedure for multi-crystal data collection (Guo et al., 2018; Takemaru et al., 2020) and successfully extended the method to native SAD phasing (Guo et al., 2019). In Step 1, each single crystal is indexed and integrated using DIALS (Winter et al., 2018), and a unit-cell clustering scheme is used to produce a reference dataset. In Step 2, based on the relative correlation coefficient comparing data from a single-crystal dataset to the reference dataset, one can identify a refined selection of crystals and images from which the final merged datasets can be combined. Step 3 includes an iterative crystal and image rejection filter to generate a sorted succession of merged datasets at varied levels of accepted accumulated dose. Eventually, one can evaluate the merged datasets from Step 3 by measures of data quality and structural analysis.

\subsection{Serial crystallography}

FMX supports the two main sample-delivery methods to bring new crystal targets rapidly into the beam - goniometerbased raster scanning and high-viscosity extrusion in a jet.

7.5.1. Raster scanning serial crystallography. To maximize sample throughput for raster scanning serial crystallography, we have developed a high-speed high-precision goniometer based on a unique $X Y Z$ piezo positioner with sub-100 nm raster scanning precision at over 40 raster lines per second scanning frequency (Gao et al., 2018). We have obtained highquality diffraction datasets by serial crystallography up to the Eiger X 16M's maximum frame rate of $750 \mathrm{~Hz}$ (using the $4 \mathrm{M}$ central region) and using the full flux of the beamline. The collection parameters were tuned to obtain multiple rotation diffraction images per crystal, thus requiring fewer crystals for a complete dataset. Multiple raster scans were performed sequentially over the region of interest, with a total shutteropen time of $18 \mathrm{~s}$ for a complete dataset selectively combined from over 400 partial datasets (Gao et al., 2018). This is a speed increase of around two orders of magnitude over earlier implementations (Gati et al., 2014; Coquelle et al., 2015), reducing data collection times from hours to under a minute, and it does not require loading a fixed target matrix sample holder (Roedig et al., 2015; Mueller et al., 2015; Davy et al., 2019).

To process the multi-crystal rotation raster data, we developed a Python-scripted workflow, called WYpeline, which employs mainly DOZOR (Zander et al., 2015) and XDS (Kabsch, 2010). Each partial dataset, determined by DOZOR, is separately indexed and integrated in $X D S$. A clustering based on unit-cell sizes and a hierarchical clustering of the correlation coefficients from XSCALE (Giordano et al., 2012; Santoni et al., 2017) yield the final merged dataset. To demonstrate the advantages of the versatile data collection schemes above, we compiled examples for these use cases on typical samples in collaboration with the work group of Sandra Gabelli (Miller et al., 2019).

7.5.2. Serial crystallography with a high-viscosity extrusion injector. We have partnered with the group of John Spence
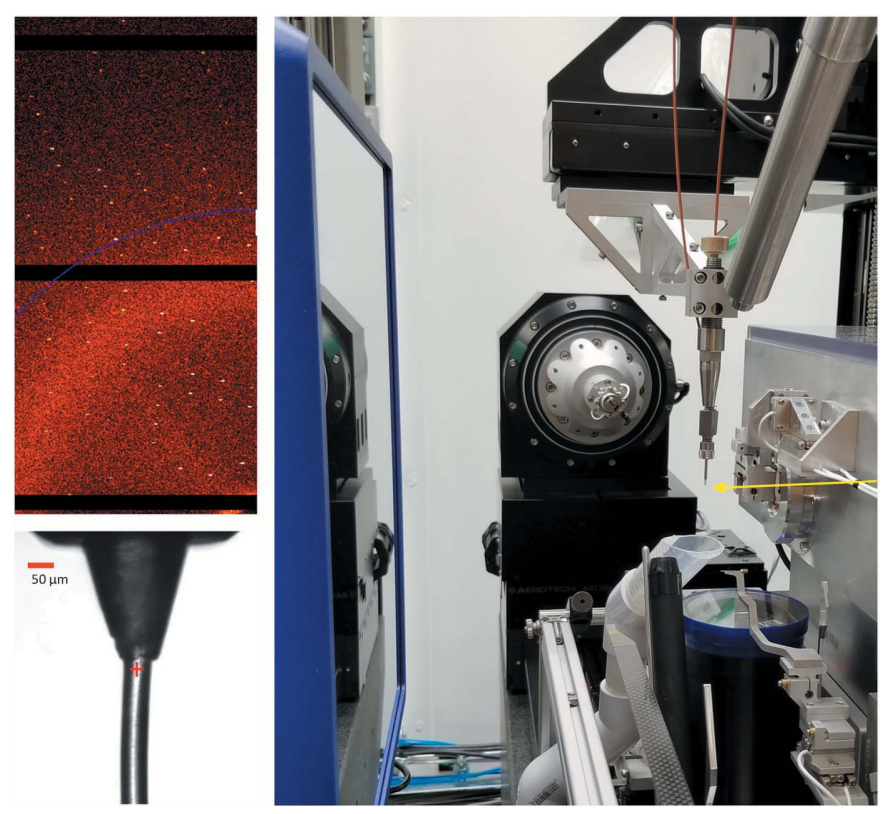

Figure 11

Serial crystallography with an LCP jet on FMX. (Right) The highviscosity extrusion injector mounted on the secondary goniometer on the FMX beamline. The beam (yellow arrow) hits the LCP stream ejected downwards from the injector nozzle. (Bottom left) A view of the injector nozzle tip in the sample microscope. The nozzle is aligned so the beam (red cross) hits the viscous jet just underneath the nozzle. (Top left) Still diffraction images are recorded on the Eiger X 16M detector.

(Arizona State University, USA) to operate a high-viscosity extrusion injector to enable data collection of microcrystals in LCP and other compatible viscous media. We injected microcrystal $(5-10 \mu \mathrm{m})$ suspensions to the intersection with the X-ray beam (Botha et al., in preparation) using the highviscosity extrusion injector (Weierstall et al., 2014) with a 20 $50 \mu \mathrm{m}$ ID nozzle and a minimal flow speed of $100-300 \mu \mathrm{m} \mathrm{s}^{-1}$. A $20 \mu \mathrm{l}$ reservoir of crystal slurry provides $4-10 \mathrm{~h}$ of injector flow for data collection, depending on the flow rate. When in operation, the injector is mounted on the secondary goniometer to align the injector downwards perpendicular to the X-ray beam path (Fig. 11) and typically 10000-100000 single diffraction images will be recorded at up to $750 \mathrm{~Hz}$ while the microcrystals are passing through the X-ray beam. To filter the data, Cheetah (Barty et al., 2014) or DOZOR (Zander et al., 2015) identify the images containing diffraction patterns, and these are processed with CrystFEL (White et al., 2016).

The main appeal of the jet for the synchrotron MX user base is the optimized workflow the jet system provides for LCP-grown microcrystals.

\section{Conclusion}

The Frontier Microfocus Macromolecular Crystallography beamline FMX at NSLS-II provides an ultra-brilliant beam to push the boundaries of microfocus crystallography. In its first two years of general user operation, we have established a broad range of sample-delivery and data collection modes that can be tailored to the crystallographic and structural biology problems our users bring to the beamline. 


\section{Acknowledgements}

We would like to thank Kenneth Evans-Lutterodt for providing us the $\mathrm{Cr}$ nanowire for the beam diagnostics, and our collaborators from Arizona State University, Jose Martin Garcia, Sabine Botha, Nadia Zatsepin, Hao Hu, Uwe Weierstall and John Spence, for designing, setting up and help supporting the LCP-jet sample delivery and data processing and for co-organizing the 2019 jet workshop. We would further like to thank Andreas Biermanns-Foeth, Michael Hoffmann, Sebastian Szillat and Christian Piel from Research Instruments, Germany, and Andreas Schacht, Timm Waterstradt and Wolfgang Diete from Axilon AG, Germany, for design and fabrication, and for their help and advice during the commissioning of the photon delivery system. We are also grateful to Tung-Chung Mou and Stephen Sprang for the figure of Ric 8A, and to Alla Peselis and Alexander Serganov for the crystal picture and figure of the ppGpp riboswitch.

\section{Funding information}

This research was supported by BNL LDRD program FY16006, and uses resources of the NSLS-II, which is supported by the US Department of Energy (grant No. BES-FWP-PS001). The FMX beamline is further supported by the National Institutes of Health (NIH) [National Institute of General Medical Sciences (NIGMS) grant No. 1P30GM133893] and the Department of Energy, Office of Biological and Environmental Research (BER) (grant No. BER-BO 070).

\section{References}

Adams, P. D., Afonine, P. V., Bunkóczi, G., Chen, V. B., Davis, I. W., Echols, N., Headd, J. J., Hung, L.-W., Kapral, G. J., GrosseKunstleve, R. W., McCoy, A. J., Moriarty, N. W., Oeffner, R., Read, R. J., Richardson, D. C., Richardson, J. S., Terwilliger, T. C. \& Zwart, P. H. (2010). Acta Cryst. D66, 213-221.

Aishima, J., Owen, R. L., Axford, D., Shepherd, E., Winter, G., Levik, K., Gibbons, P., Ashton, A. \& Evans, G. (2010). Acta Cryst. D66, 1032-1035.

Allan, D. R., Collins, S. P., Evans, G., Hall, D., McAuley, K., Owen, R. L., Sorensen, T., Tang, C. C., von Delft, F., Wagner, A. \& Wilhelm, H. (2015). Eur. Phys. J. Plus, 130, 56.

Arkilic, A., Allan, D. B., Caswell, T. A., Li, L., Lauer, K. \& Abeykoon, S. (2017). Synchrotron Radiat. News, 30(2), 44-45.

Axford, D., Foadi, J., Hu, N.-J., Choudhury, H. G., Iwata, S., Beis, K., Evans, G. \& Alguel, Y. (2015). Acta Cryst. D71, 1228-1237.

Barty, A., Kirian, R. A., Maia, F. R. N. C., Hantke, M., Yoon, C. H., White, T. A. \& Chapman, H. (2014). J. Appl. Cryst. 47, 1118-1131.

Berman, L. E., Allaire, M., Chance, M. R., Hendrickson, W. A., Héroux, A., Jakoncic, J., Liu, Q., Orville, A. M., Robinson, H. H., Schneider, D. K., Shi, W., Soares, A. S., Stojanoff, V., Stoner-Ma, D. \& Sweet, R. M. (2011). Nucl. Instrum. Methods Phys. Res. A, 649, 131-135.

Bhogadi, D. K., Andi, B. A., Berman, L., Carlucci-Dayton, M., Fuchs, M. R., Jakoncic, J., Langdon, T., Lara, J., Martins, B. S., McSweeney, S., Myers, S. F., Schneider, D. K. \& Sweet, R. M. (2017). Proceedings of the 9th Mechanical Engineering Design of Synchrotron Radiation Equipment and Instrumentation (MEDSI2016), 11-16 September 2016, Barcelona, Spain, pp. 363-366. Geneva: JACoW.

Bowler, M. W., Guijarro, M., Petitdemange, S., Baker, I., Svensson, O., Burghammer, M., Mueller-Dieckmann, C., Gordon, E. J., Flot,
D., McSweeney, S. M. \& Leonard, G. A. (2010). Acta Cryst. D66, 855-864.

Burkhardt, A., Pakendorf, T., Reime, B., Meyer, J., Fischer, P., Stübe, N., Panneerselvam, S., Lorbeer, O., Stachnik, K., Warmer, M., Rödig, P., Göries, D. \& Meents, A. (2016). Eur. Phys. J. Plus, 131, 56.

Cherezov, V., Hanson, M. A., Griffith, M. T., Hilgart, M. C., Sanishvili, R., Nagarajan, V., Stepanov, S., Fischetti, R. F., Kuhn, P. \& Stevens, R. C. (2009). J. R. Soc. Interface, 6, s587.

Cianci, M., Bourenkov, G., Pompidor, G., Karpics, I., Kallio, J., Bento, I., Roessle, M., Cipriani, F., Fiedler, S. \& Schneider, T. R. (2017). J. Synchrotron Rad. 24, 323-332.

Cipriani, F., Felisaz, F., Launer, L., Aksoy, J.-S., Caserotto, H., Cusack, S., Dallery, M., di-Chiaro, F., Guijarro, M., Huet, J., Larsen, S., Lentini, M., McCarthy, J., McSweeney, S., Ravelli, R., Renier, M., Taffut, C., Thompson, A., Leonard, G. A. \& Walsh, M. A. (2006). Acta Cryst. D62, 1251-1259.

Clausen, M. R., Gerke, C. H., Moeller, M., Rickens, H. R. \& Hatje, J. (2007). Proceedings of the 11th International Conference on Accelerator and Large Experimental Physics Control Systems (ICALEPCS2007), 15-19 October 2007, Knoxville, Tennessee, USA, pp. 37-39. MOPB03.

Coquelle, N., Brewster, A. S., Kapp, U., Shilova, A., Weinhausen, B., Burghammer, M. \& Colletier, J.-P. (2015). Acta Cryst. D71, 11841196.

Cowan, J. A. \& Nave, C. (2008). J. Synchrotron Rad. 15, 458-462.

Davy, B., Axford, D., Beale, J. H., Butryn, A., Docker, P., Ebrahim, A., Leen, G., Orville, A. M., Owen, R. L. \& Aller, P. (2019). J. Synchrotron Rad. 26, 1820-1825.

Delagenière, S., Brenchereau, P., Launer, L., Ashton, A. W., Leal, R., Veyrier, S., Gabadinho, J., Gordon, E. J., Jones, S. D., Levik, K. E., McSweeney, S. M., Monaco, S., Nanao, M., Spruce, D., Svensson, O., Walsh, M. A. \& Leonard, G. A. (2011). Bioinformatics, 27, 31863192.

Dickerson, J. L. \& Garman, E. F. (2019). J. Synchrotron Rad. 26, 922 930.

Ebrahim, A., Appleby, M. V., Axford, D., Beale, J., Moreno-Chicano, T., Sherrell, D. A., Strange, R. W., Hough, M. A. \& Owen, R. L. (2019). Acta Cryst. D75, 151-159.

Evans, G. \& Pettifer, R. F. (2001). J. Appl. Cryst. 34, 82-86.

Evans, P. (2006). Acta Cryst. D62, 72-82.

Evans, P. R. \& Murshudov, G. N. (2013). Acta Cryst. D69, 1204-1214.

Finfrock, Y. Z., Stern, E. A., Alkire, R. W., Kas, J. J., Evans-Lutterodt, K., Stein, A., Duke, N., Lazarski, K. \& Joachimiak, A. (2013). Acta Cryst. D69, 1463-1469.

Fischetti, R. F., Yoder, D., Xu, S., Makarov, O., Ogata, C. \& Smith, J. L. (2013). J. Phys. Conf. Ser. 425, 012006.

Fisher, S. J., Levik, K. E., Williams, M. A., Ashton, A. W. \& McAuley, K. E. (2015). J. Appl. Cryst. 48, 927-932.

Flot, D., Mairs, T., Giraud, T., Guijarro, M., Lesourd, M., Rey, V., van Brussel, D., Morawe, C., Borel, C., Hignette, O., Chavanne, J., Nurizzo, D., McSweeney, S. \& Mitchell, E. (2010). J. Synchrotron Rad. 17, 107-118.

Fuchs, M. R., Bhogadi, D. K., Jakoncic, J., Myers, S., Sweet, R. M., Berman, L. E., Skinner, J., Idir, M., Chubar, O., McSweeney, S. \& Schneider, D. K. (2016). AIP Conf. Proc., 1741, 030006.

Fuchs, M. R., Sweet, R. M., Berman, L. E., Hendrickson, W. A., Chubar, O., Canestrari, N., Idir, M., Yang, L. \& Schneider, D. K. (2014). J. Phys. Conf. Ser. 493, 012021.

Gabadinho, J., Beteva, A., Guijarro, M., Rey-Bakaikoa, V., Spruce, D., Bowler, M. W., Brockhauser, S., Flot, D., Gordon, E. J., Hall, D. R., Lavault, B., McCarthy, A. A., McCarthy, J., Mitchell, E., Monaco, S., Mueller-Dieckmann, C., Nurizzo, D., Ravelli, R. B. G., Thibault, X., Walsh, M. A., Leonard, G. A. \& McSweeney, S. M. (2010). J. Synchrotron Rad. 17, 700-707.

Gao, Y., Xu, W., Shi, W., Soares, A., Jakoncic, J., Myers, S., Martins, B., Skinner, J., Liu, Q., Bernstein, H., McSweeney, S., Nazaretski, E. \& Fuchs, M. R. (2018). J. Synchrotron Rad. 25, 1362-1370. 
Gati, C., Bourenkov, G., Klinge, M., Rehders, D., Stellato, F., Oberthür, D., Yefanov, O., Sommer, B. P., Mogk, S., Duszenko, M., Betzel, C., Schneider, T. R., Chapman, H. N. \& Redecke, L. (2014). IUCrJ, 1, 87-94.

Giordano, R., Leal, R. M. F., Bourenkov, G. P., McSweeney, S. \& Popov, A. N. (2012). Acta Cryst. D68, 649-658.

Goetze, K. \& Lienert, U. (2009). A Galvanometer Based Fast Shutter. Poster presented at the 10th International Conference on Synchrotron Radiation Instrumentation SRI2009, 27 September-2 October 2009, Melbourne, Australia.

Grosse-Kunstleve, R. W., Sauter, N. K., Moriarty, N. W. \& Adams, P. D. (2002). J. Appl. Cryst. 35, 126-136.

Guo, G., Fuchs, M. R., Shi, W., Skinner, J., Berman, E., Ogata, C. M., Hendrickson, W. A., McSweeney, S. \& Liu, Q. (2018). IUCrJ, 5, 238-246.

Guo, G., Zhu, P., Fuchs, M. R., Shi, W., Andi, B., Gao, Y., Hendrickson, W. A., McSweeney, S. \& Liu, Q. (2019). IUCrJ, 6, $532-542$.

Hasegawa, K., Shimizu, N., Okumura, H., Mizuno, N., Baba, S., Hirata, K., Takeuchi, T., Yamazaki, H., Senba, Y., Ohashi, H., Yamamoto, M. \& Kumasaka, T. (2013). J. Synchrotron Rad. 20, 910-913.

Hidaka, Y., Caracappa, A., Hu, Y., Podobedov, B., Smith, R., Tian, Y. \& Wang, G. (2019). Proceedings of IPAC2019, 19-24 May 2019, Melbourne, Australia, pp. 4070-4073. Geneva: JACoW.

Hirata, K., Kawano, Y., Ueno, G., Hashimoto, K., Murakami, H., Hasegawa, K., Hikima, T., Kumasaka, T. \& Yamamoto, M. (2013). J. Phys. Conf. Ser. 425, 012002.

Holton, J. M., Classen, S., Frankel, K. A. \& Tainer, J. A. (2014). FEBS J. 281, 4046-4060.

Huang, C.-Y., Olieric, V., Ma, P., Howe, N., Vogeley, L., Liu, X., Warshamanage, R., Weinert, T., Panepucci, E., Kobilka, B., Diederichs, K., Wang, M. \& Caffrey, M. (2016). Acta Cryst. D72, 93-112.

Ilinski, P. (2013). J. Phys. Conf. Ser. 425, 042006.

Incardona, M.-F., Bourenkov, G. P., Levik, K., Pieritz, R. A., Popov, A. N. \& Svensson, O. (2009). J. Synchrotron Rad. 16, 872-879.

Kabsch, W. (2010). Acta Cryst. D66, 125-132.

Keedy, D. A., Kenner, L. R., Warkentin, M., Woldeyes, R. A., Hopkins, J. B., Thompson, M. C., Brewster, A. S., Van Benschoten, A. H., Baxter, E. L., Uervirojnangkoorn, M., McPhillips, S. E., Song, J., Alonso-Mori, R., Holton, J. M., Weis, W. I., Brunger, A. T., Soltis, S. M., Lemke, H., Gonzalez, A., Sauter, N. K., Cohen, A. E., van den Bedem, H., Thorne, R. E. \& Fraser, J. S. (2015). eLife, 4, $\mathrm{e} 07574$.

Keister, J. W., Cibik, L., Schreiber, S. \& Krumrey, M. (2018). J. Synchrotron Rad. 25, 407-412.

Lazo, E. O., Bernstein, H. J., Bhogadi, D., Fuchs, M. R., Guichard, N., McSweeney, S., Myers, S., Qian, K., Schneider, D., Shea-McCarthy, G., Sweet, R., Yang, L. \& Jakoncic, J. (2021). J. Synchrotron Rad. To be submitted.

Lyubimov, A. Y., Uervirojnangkoorn, M., Zeldin, O. B., Zhou, Q., Zhao, M., Brewster, A. S., Michels-Clark, T., Holton, J. M., Sauter, N. K., Weis, W. I. \& Brunger, A. T. (2016). eLife, 5, e18740.

Marman, H., Darmanin, C. \& Abbey, B. (2018). Crystals, 8, 267.

McClelland, L. J., Zhang, K., Mou, T.-C., Johnston, J., Yates-Hansen, C., Li, S., Thomas, C. J., Doukov, T. I., Triest, S., Wohlkonig, A., Tall, G. G., Steyaert, J., Chiu, W. \& Sprang, S. R. (2020). Nat. Commun. 11, 1077.

Miller, M. S., Maheshwari, S., Shi, W., Gao, Y., Chu, N., Soares, A. S., Cole, P. A., Amzel, L. M., Fuchs, M. R., Jakoncic, J. \& Gabelli, S. B. (2019). Molecules, 24, 496.

Mueller, C., Marx, A., Epp, S. W., Zhong, Y., Kuo, A., Balo, A. R., Soman, J., Schotte, F., Lemke, H. T., Owen, R. L., Pai, E. F., Pearson, A. R., Olson, J. S., Anfinrud, P. A., Ernst, O. P. \& Dwayne Miller, R. J. (2015). Struct. Dyn. 2, 054302.

Mueller-Dieckmann, C., Bowler, M. W., Carpentier, P., Flot, D., McCarthy, A. A., Nanao, M. H., Nurizzo, D., Pernot, P., Popov, A.,
Round, A., Royant, A., de Sanctis, D., von Stetten, D. \& Leonard, G. A. (2015). Eur. Phys. J. Plus, 130, 70.

Nave, C. (2014). J. Synchrotron Rad. 21, 537-546.

NSLS-II (2019). NSLS-II software documentation, https://nsls-ii. github.io/.

Otwinowski, Z. \& Minor, W. (1997). Methods in Enzymology, Vol. 276, Macromolecular Crystallography, Part A, edited by C. W. Carter Jr \& R. M. Sweet, pp. 307-326. New York: Academic Press.

Owen, R. L., Axford, D., Sherrell, D. A., Kuo, A., Ernst, O. P., Schulz, E. C., Miller, R. J. D. \& Mueller-Werkmeister, H. M. (2017). Acta Cryst. D73, 373-378.

Owen, R. L., Juanhuix, J. \& Fuchs, M. (2016). Arch. Biochem. Biophys. 602, 21-31.

Owen, R. L., Rudiño-Piñera, E. \& Garman, E. F. (2006). Proc. Nat. Acad. Sci. USA, 103, 4912-4917.

Pape, T. \& Schneider, T. R. (2004). J. Appl. Cryst. 37, 843-844.

Papp, G., Rossi, C., Janocha, R., Sorez, C., Lopez-Marrero, M., Astruc, A., McCarthy, A., Belrhali, H., Bowler, M. W. \& Cipriani, F. (2017). Acta Cryst. D73, 829-840.

Peselis, A. \& Serganov, A. (2018). Nat. Chem. Biol. 14, 887-894.

Riekel, C., Burghammer, M. \& Davies, R. (2010). IOP Conf. Ser. Mater. Sci. 14, 012013.

Roedig, P., Vartiainen, I., Duman, R., Panneerselvam, S., Stübe, N., Lorbeer, O., Warmer, M., Sutton, G., Stuart, D. I., Weckert, E., David, C., Wagner, A. \& Meents, A. (2015). Sci. Rep. 5, 10451.

Russi, S., González, A., Kenner, L. R., Keedy, D. A., Fraser, J. S. \& van den Bedem, H. (2017). J. Synchrotron Rad. 24, 73-82.

Sanishvili, R., Yoder, D. W., Pothineni, S. B., Rosenbaum, G., Xu, S., Vogt, S., Stepanov, S., Makarov, O. A., Corcoran, S., Benn, R., Nagarajan, V., Smith, J. L. \& Fischetti, R. F. (2011). Proc. Natl Acad. Sci. USA, 108, 6127-6132.

Santoni, G., Zander, U., Mueller-Dieckmann, C., Leonard, G. \& Popov, A. (2017). J. Appl. Cryst. 50, 1844-1851.

Sauter, N. K., Hattne, J., Grosse-Kunstleve, R. W. \& Echols, N. (2013). Acta Cryst. D69, 1274-1282.

Schneider, D. K., Berman, L. E., Chubar, O., Hendrickson, W. A., Hulbert, S. L., Lucas, M., Sweet, R. M. \& Yang, L. (2013). J. Phys. Conf. Ser. 425, 012003.

Schulze-Briese, C., Tomizaki, T., Pradervand, C., Schneider, R., Danousch, M., Portmann, W., Chen, Q., Ingold, G., Rossetti, D., Frauenfelder, B., Zumbach, C., Hottinger, P., Brönnimann, C. \& Eikenberry, E. F. (2002). Swiss Light Source Scientific Report 2001, Vol. VII, pp 54-55. Paul Scherrer Institute, Villigen, Switzerland.

Sheldrick, G. M. (2015). Acta Cryst. C71, 3-8.

Smaluk, V., Li, Y., Hidaka, Y., Tanabe, T., Chubar, O., Wiegart, L., Blednykh, A., Bacha, B. \& Shaftan, T. (2019). Phys. Rev. Accel. Beams, 22, 124001.

Smith, J. L., Fischetti, R. F. \& Yamamoto, M. (2012). Curr. Opin. Struct. Biol. 22, 602-612.

Soltis, S. M., Cohen, A. E., Deacon, A., Eriksson, T., González, A., McPhillips, S., Chui, H., Dunten, P., Hollenbeck, M., Mathews, I., Miller, M., Moorhead, P., Phizackerley, R. P., Smith, C., Song, J., van dem Bedem, H., Ellis, P., Kuhn, P., McPhillips, T., Sauter, N., Sharp, K., Tsyba, I. \& Wolf, G. (2008). Acta Cryst. D64, 12101221.

Song, J., Mathew, D., Jacob, S. A., Corbett, L., Moorhead, P. \& Soltis, S. M. (2007). J. Synchrotron Rad. 14, 191-195.

Stetten, D. von, Carpentier, P., Flot, D., Beteva, A., Caserotto, H., Dobias, F., Guijarro, M., Giraud, T., Lentini, M., McSweeney, S., Royant, A., Petitdemange, S., Sinoir, J., Surr, J., Svensson, O., Theveneau, P., Leonard, G. A. \& Mueller-Dieckmann, C. (2020). J. Synchrotron Rad. 27, 844-851.

Sutter, J. P., Alcock, S. G. \& Sawhney, K. (2013). Nucl. Instrum. Methods Phys. Res. A, 710, 72-77.

Sutter, J. P., Alcock, S. G. \& Sawhney, K. J. S. (2011). Proc. SPIE, 8139, 813906-813911.

Takemaru, L., Guo, G., Zhu, P., Hendrickson, W. A., McSweeney, S. \& Liu, Q. (2020). J. Appl. Cryst. 53, 277-281. 
Ursby, T., Åhnberg, K., Appio, R., Aurelius, O., Barczyk, A., Bartalesi, A., Bjelčić, M., Bolmsten, F., Cerenius, Y., Doak, R. B., Eguiraun, M., Eriksson, T., Friel, R. J., Gorgisyan, I., Gross, A., Haghighat, V., Hennies, F., Jagudin, E., Nor. Jensen, B., Jeppsson, T., Kloos, M., Lidon-Simon, J., de Lima, G. M. A., Lizatovic, R., Lundin, M., Milan-Otero, A., Milas, M., Nan, J., Nardella, A., Rosborg, A., Shilova, A., Shoeman, R. L., Siewert, F., Sondhauss, P., Talibov, V. O., Tarawneh, H., Thånell, J., Thunnissen, M., Unge, J., Ward, C., Gonzalez, A. \& Mueller, U. (2020). J. Synchrotron Rad. 27, 1415-1429.

Warkentin, M., Hopkins, J. B., Badeau, R., Mulichak, A. M., Keefe, L. J. \& Thorne, R. E. (2013). J. Synchrotron Rad. 20, 7-13.

Weierstall, U., James, D., Wang, C., White, T. A., Wang, D., Liu, W., Spence, J. C. H., Bruce Doak, R., Nelson, G., Fromme, P., Fromme, R., Grotjohann, I., Kupitz, C., Zatsepin, N. A., Liu, H., Basu, S., Wacker, D., Won Han, G., Katritch, V., Boutet, S., Messerschmidt, M., Williams, G. J., Koglin, J. E., Marvin Seibert, M., Klinker, M., Gati, C., Shoeman, R. L., Barty, A., Chapman, H. N., Kirian, R. A., Beyerlein, K. R., Stevens, R. C., Li, D., Shah, S. T. A., Howe, N., Caffrey, M. \& Cherezov, V. (2014). Nat. Commun. 5, 3309.

White, T. A., Mariani, V., Brehm, W., Yefanov, O., Barty, A., Beyerlein, K. R., Chervinskii, F., Galli, L., Gati, C., Nakane, T.,
Tolstikova, A., Yamashita, K., Yoon, C. H., Diederichs, K. \& Chapman, H. N. (2016). J. Appl. Cryst. 49, 680-689.

Winn, M. D., Ballard, C. C., Cowtan, K. D., Dodson, E. J., Emsley, P., Evans, P. R., Keegan, R. M., Krissinel, E. B., Leslie, A. G. W., McCoy, A., McNicholas, S. J., Murshudov, G. N., Pannu, N. S. Potterton, E. A., Powell, H. R., Read, R. J., Vagin, A. \& Wilson, K. S. (2011). Acta Cryst. D67, 235-242.

Winter, G. \& McAuley, K. E. (2011). Methods, 55, 81-93.

Winter, G., Waterman, D. G., Parkhurst, J. M., Brewster, A. S., Gildea, R. J., Gerstel, M., Fuentes-Montero, L., Vollmar, M., MichelsClark, T., Young, I. D., Sauter, N. K. \& Evans, G. (2018). Acta Cryst. D74, 85-97.

Yamamoto, M., Hirata, K., Yamashita, K., Hasegawa, K., Ueno, G., Ago, H. \& Kumasaka, T. (2017). IUCrJ, 4, 529-539.

Yang, L., Antonelli, S., Chodankar, S., Byrnes, J., Lazo, E. \& Qian, K. (2020). J. Synchrotron Rad. 27, 804-812.

Zander, U., Bourenkov, G., Popov, A. N., de Sanctis, D., Svensson, O., McCarthy, A. A., Round, E., Gordeliy, V., Mueller-Dieckmann, C. \& Leonard, G. A. (2015). Acta Cryst. D71, 2328-2343.

Zeng, B., Mou, T.-C., Doukov, T. I., Steiner, A., Yu, W., PapasergiScott, M., Tall, G. G., Hagn, F. \& Sprang, S. R. (2019). Structure, 27, 1137-1147.e5. 